OPEN ACCESS

Edited by:

Theodore R. Cummins, Purdue University Indianapolis, United States

Reviewed by:

Oscar Moran,

Biophysics Institute, National

Research Council (CNR), Italy

Hai Minh Nguyen,

University of California, Davis, United States

*Correspondence:

Karl Josef Föhr

karl.foehr@uniklinik-ulm.de

Specialty section: This article was submitted to Pharmacology of Ion Channels and

Channelopathies,

a section of the journal

Frontiers in Pharmacology

Received: 08 July 2021 Accepted: 15 September 2021

Published: 21 October 2021

Citation:

Fuchs E, Messerer DAC, Karpel-Massler G, Fauler M, Zimmer T, Jungwirth B and Föhr KJ (2021) Block of Voltage-Gated Sodium Channels as a Potential Novel Anti-cancer Mechanism of TIC10. Front. Pharmacol. 12:737637. doi: 10.3389/fphar.2021.737637

\section{Block of Voltage-Gated Sodium Channels as a Potential Novel Anti-cancer Mechanism of TIC10}

Eva Fuchs ${ }^{1}$, David Alexander Christian Messerer ${ }^{1}$, Georg Karpel-Massler ${ }^{2}$, Michael Fauler ${ }^{3}$, Thomas Zimmer ${ }^{4}$, Bettina Jungwirth ${ }^{1}$ and Karl Josef Föhr ${ }^{1 *}$

${ }^{1}$ Department of Anesthesiology and Intensive Care Medicine, University Hospital of UIm, Ulm, Germany, ${ }^{2}$ Department of Neurological Surgery, University Hospital of Ulm, Ulm, Germany, ${ }^{3}$ Institute of General Physiology, University of Ulm, Ulm, Germany, ${ }^{4}$ Institute of Physiology, University Hospital of Jena, Jena, Germany

Background: Tumor therapeutics are aimed to affect tumor cells selectively while sparing healthy ones. For this purpose, a huge variety of different drugs are in use. Recently, also blockers of voltage-gated sodium channels (VGSCs) have been recognized to possess potentially beneficial effects in tumor therapy. As these channels are a frequent target of numerous drugs, we hypothesized that currently used tumor therapeutics might have the potential to block VGSCs in addition to their classical anti-cancer activity. In the present work, we have analyzed the imipridone $\mathrm{TIC} 10$, which belongs to a novel class of anticancer compounds, for its potency to interact with VGSCs.

Methods: Electrophysiological experiments were performed by means of the patchclamp technique using heterologously expressed human heart muscle sodium channels (hNav1.5), which are among the most common subtypes of VGSCs occurring in tumor cells.

Results: TIC10 angular inhibited the $\mathrm{hNa}_{v} 1.5$ channel in a state- but not use-dependent manner. The affinity for the resting state was weak with an extrapolated $\mathrm{K}_{\mathrm{r}}$ of about $600 \mu \mathrm{M}$. TIC10 most probably did not interact with fast inactivation. In protocols for slow inactivation, a half-maximal inhibition occurred around $2 \mu \mathrm{M}$. This observation was confirmed by kinetic studies indicating that the interaction occurred with a slow time constant. Furthermore, TIC10 also interacted with the open channel with an affinity of approximately $4 \mu \mathrm{M}$. The binding site for local anesthetics or a closely related site is suggested as a possible target as the affinity for the well-characterized F1760K mutant was reduced more than 20 -fold compared to wild type. Among the analyzed derivatives, ONC212 was similarly effective as TIC10 angular, while TIC10 linear more selectively interacted with the different states.

Conclusion: The inhibition of VGSCs at low micromolar concentrations might add to the anti-tumor properties of TIC10.

Keywords: TIC10, ONC201, voltage-gated sodium channel, drug repurposing, tumor therapy 


\section{INTRODUCTION}

TIC10, also known as ONC201 or NSC350625, belongs to a novel class of anti-cancer compounds called imipridones (Wagner et al., 2017). It operates independently from p53 and provides antiproliferative and pro-apoptotic effects against a broad range of tumors, including hematological malignancies, glioblastoma, colorectal cancer or cancer stem cells (Allen et al., 2016). Altogether, a favorable side effect profile was observed in clinical trials (Stein et al., 2019; Arrillaga-Romany et al., 2020). Meanwhile, several derivatives of TIC10 were generated with in part significantly higher potency regarding inhibition of cell growth of different types of human cancer cells. Nevertheless, TIC10 still represents the most advanced imipridone with respect to the pre-clinical and clinical investigations (Ishida et al., 2018; Ma et al., 2019). So far, different modes of action were described for TIC10: The antineoplastic activity of TIC10 was first identified in a drug screen searching for compounds that induce TRAIL. As a consequence, the name of TIC10 is derived from this function as TRAIL-inducing compound 10 (Wagner et al., 2017). Recently, the mitochondrial caseinolytic protease $\mathrm{P}$ has been proposed and identified as the direct molecular target of TIC10 (Ishizawa et al., 2019; Wang and Dougan, 2019). In addition, using a newly developed algorithm, TIC10 has been predicted to exert a highly specific interaction with dopamine $\mathrm{D}_{2}$ receptors (Kline et al., 2016). Meanwhile, the $\mathrm{D}_{2}$ antagonizing property of TIC10 has been confirmed, which in turn has been demonstrated to have anti-tumor effects (Allen et al., 2016). As the imipridones were initially developed as anti-seizure medication, voltage-gated sodium channels (VGSCs) might also be potential targets (Kline et al., 2016; Pruss et al., 2020). Therefore, it is tempting to speculate that a link to a possible anti-tumor activity exists, as blockers of VGSCs are known to affect tumor metastasis (Djamgoz et al., 2019).

VGSCs are composed from one out of nine different pore forming $\alpha$-subunits which are associated with no, one or two out of four auxiliary B-subunits (Catterall et al., 2005; Patino and Isom, 2010). The $\alpha$-subunits are designated as $\mathrm{Na}_{\mathrm{v}} 1.1$ to $\mathrm{Na}_{\mathrm{v}} 1.9$ according to their phylogeny. Other diversity arises from alternative splicing of mRNA and post-translational modifications. The distribution of the different subunits is tissue specific. $\mathrm{Na}_{\mathrm{v}} 1.4$ and $\mathrm{Na}_{\mathrm{v}} 1.5$ are predominantly found in skeletal and heart muscle cells, whereas $\mathrm{Na}_{\mathrm{v}} 1.1$ to $\mathrm{Na}_{\mathrm{v}} 1.3$ and $\mathrm{Na}_{\mathrm{v}} 1.6$ to $\mathrm{Na}_{\mathrm{v}} 1.9$ are found in neurons. According to pharmacological parameters the individual channels are classified as TTX-sensitive or TTX-resistant, depending on the concentration of tetrodotoxin (TTX) which is required for their blockage. Further classifications are based on different subunitspecific electrophysiological properties (Theile and Cummins, 2011).

During a screening of different anti-cancer agents, we identified TIC10/ONC201 as a potent blocker of VGSCs. In the present work, we investigated the potential of TIC10 to block VGSCs, which might add a supplementary effect on therapy. So far, almost all VGSC subtypes have been detected in different tumor entities (Roger et al., 2015). Most prevalent are the $\mathrm{Na}_{\mathrm{v}} 1.7$ and the $\mathrm{Na}_{\mathrm{v}} 1.5$ subtype. The latter was found to be present in a huge variety of diverse malignancies including breast cancer, colon cancer, ovarian cancer, melanoma, astrocytoma, or neuroblastoma (Djamgoz et al., 2019). Therefore, we performed the underlying experiments with the VGSC preferentially expressed in the heart muscle $\left(\mathrm{hNa}_{\mathrm{v}} 1.5\right)$. This study reports about details regarding the mechanism of the underlying interaction.

\section{METHODS}

\section{Cell Culture}

The tsA201 cell line is a transformed human kidney 293 (HEK293) cell line stably expressing an SV40 temperaturesensitive $\mathrm{T}$ antigen (Sigma-Aldrich no. 85120602). TsA201 cells were cultured at $37^{\circ} \mathrm{C}$ in a humidified atmosphere at $95 \%$ air and $5 \% \mathrm{CO}_{2}$ in MEM (minimum essential medium) supplemented with $50 \mathrm{U} / \mathrm{ml}$ penicillin, $50 \mu \mathrm{g} / \mathrm{ml}$ streptomycin (Gibco, Eggenstein, Germany), $2 \mathrm{mM}$ L-glutamine (Boehringer, Mannheim, Germany), and 10\% fetal calf serum (Gibco). The cells were grown on polyornithine-coated culture dishes to $40 \%$ confluency and transfected using the TransFectin LipidReagent kit (Bio-Rad, München, Germany). The construction of the plasmid pTSV40G-hNa 1.5 encoding wild-type $\mathrm{hNa}_{\mathrm{v}} 1.5$ was described previously (Walzik et al., 2011). This plasmid allows for a simplified selection of transfected cells as EGFP is simultaneously produced from a separate expression cassette. Mutant channels $\left(\mathrm{hNa}_{\mathrm{v}} 1.5 \_\mathrm{WCW}\right.$ and $\left.\mathrm{hNa}_{\mathrm{v}} 1.5 \_\mathrm{WCW} \_\mathrm{F} 1760 \mathrm{~K}\right)$ were obtained by respectively modified oligonucleotides and overlapping PCR. The PCR fragments were inserted into the pTSV40G-hNa 1.5 background using restriction sites Age/BsaBI (for WCW) and BstEII/SpeI (for F1760K), resulting in pTSV40G$\mathrm{hNa}_{\mathrm{v}} 1.5 \_\mathrm{WCW}$ and pTSV40G-hNa 1.5 _WCW_F1760K.

\section{Electrophysiology}

Electrophysiological experiments were performed as previously described (Ludolph et al., 2010; Föhr et al., 2021). Briefly, tsA201 cells were used for experiments $24-48 \mathrm{~h}$ after transfection. Membrane currents were recorded in the whole-cell recording mode using an EPC-9 amplifier and Patchmaster software (v2x73; HEKA, Lambrecht, Germany (Hamill et al., 1981)). Before recording, cells were rinsed twice with an extracellular standard solution containing (in $\mathrm{mM}$ ): $140 \mathrm{NaCl}, 5 \mathrm{KCl}, 1.5$ $\mathrm{CaCl}_{2}, 1.0 \mathrm{MgCl}_{2}, 10$ glucose and 12 HEPES (4-(2-hydroxyethyl) piperazine- 1-ethanesulfonic acid; $\mathrm{pH}$ 7.3). For establishing activation curves, the concentration of $\mathrm{NaCl}$ was reduced to $30 \mathrm{mM}$ to achieve current amplitudes in the range of $1 \mathrm{nA}$. Lacking cations were replaced equimolar by N-Methyl-Dglucamine (NMDG). Patch pipettes were drawn from borosilicate glass with tip resistances of about $2 \mathrm{M} \Omega$ when filled with (in mM): $125 \mathrm{CsF}, 10 \mathrm{NaCl}, 10$ EGTA (ethylene glycol-bis(2-aminoethylether)- $N, N, N^{\prime}, N^{\prime}$-tetraacetic acid), 10 HEPES; pH 7.2. To improve sealing, tips were briefly dipped into $2 \%$ dimethylsilane dissolved in dichloromethane.

Unless otherwise stated, the membrane potential was held at $-140 \mathrm{mV}$ from which channel activations were elicited by brief depolarizing pulses to $-20 \mathrm{mV}$ of $5 \mathrm{~ms}$ duration. In order to 


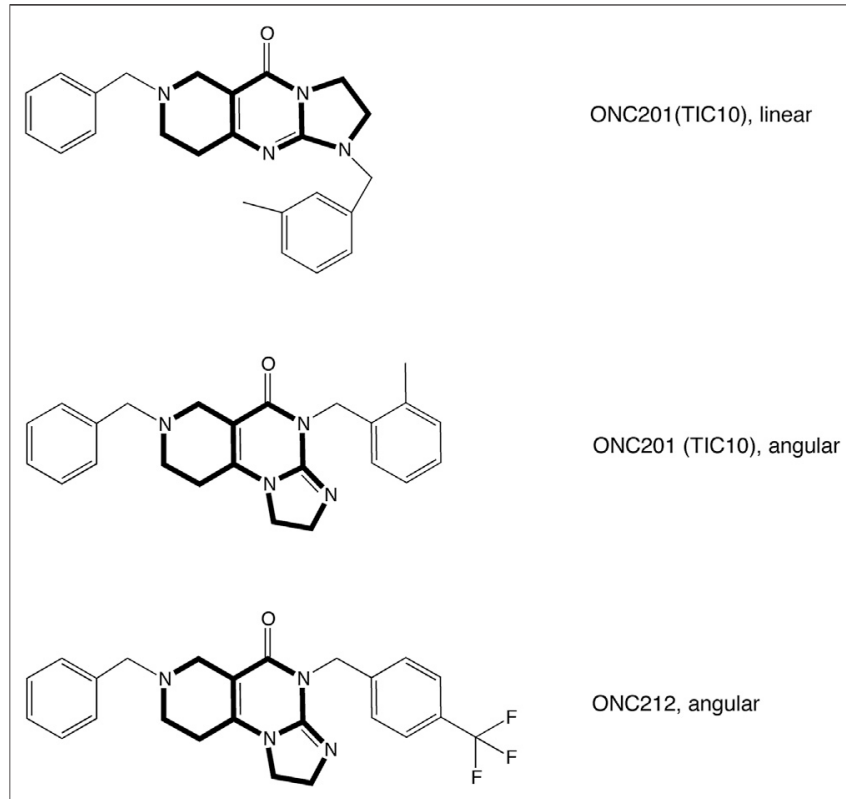

FIGURE 1 | Structural formulae of analyzed imipridones. The graph illustrates the structural formula of TIC10 also known as ONC201 and ONC212. TIC10 linear and TIC10 angular are isomeres which differ in the arrangement of the tricyclic structure.

minimize voltage errors, the series resistance was compensated up to $80 \%$. Cells with currents larger than $6 \mathrm{nA}$ were excluded from evaluation. Specific protocols are illustrated in figure legends where appropriate.

\section{Drug Application}

The medium in the dish $(1.5 \mathrm{ml})$ was continuously exchanged using a "global" bath perfusion with the inflow set to $4.5 \mathrm{ml} / \mathrm{min}$ and the outflow removing any excess fluid. Reagents were applied locally to the cells by the L/M-SPS- 8 superfusion system (List, Darmstadt, Germany). Switching between the eight channels of the superfusion system was controlled by magnetic valves. The local inlet (tip of an eight-barreled pipette) was positioned at a distance of $50-100 \mu \mathrm{m}$ upstream and the local outlet at about $300 \mu \mathrm{m}$ downstream of the patch pipette. A constant flow rate of control and test solutions $(1 \mathrm{ml} / \mathrm{min})$ was achieved by means of a pressure control system (MPCU-3, Lorenz, Göttingen, Germany). The time of solution exchange was estimated from the changes in the liquid junction potential to be about $1 \mathrm{~ms}$. If not otherwise stated, drugs were pre-applied for $20 \mathrm{~s}$ before starting the experiments.

\section{Chemicals}

Trypsin was obtained from Biochrom AG, Berlin, Germany. DNAse 1 was obtained from Invitrogen, Carlsbad, Germany; fetal calf serum was obtained from HyClone, Perbio Science, Bonn, Germany. Poly-L-ornithine was purchased from SigmaAldrich, Schnelldorf, Germany. TIC10 angular was from SigmaAldrich Chemie GmbH, Steinheim, Germany, TIC10 linear from AOBIUS, United Stated and ONC212 from Selleckchem, United Stated (Figure 1); $10 \mathrm{mM}$ stocks of these drugs were prepared with DMSO. All other chemicals were obtained from SigmaAldrich Chemie GmbH, Steinheim, Germany.

\section{Data Analysis and Statistics Concentration-Inhibition Curves}

Concentration-inhibition curves for estimating $\mathrm{K}_{\mathrm{r}}\left(I C_{50}\right.$ at $-140 \mathrm{mV}$; Figure 3), $\mathrm{K}_{\mathrm{i}}$ (Figures 8, 13, 14), $\mathrm{K}_{\mathrm{o}}$ (Figures 12, 17) or $K_{a p p}$ (Figure 9) were fitted to the Hill-equation.

$$
\frac{I_{D}}{I_{C}}=\frac{1}{1+\left(\frac{[D]}{I C_{50}}\right)^{n}}
$$

$I_{D}$ and $I_{C}$ are the current amplitudes in the presence and absence of the drug, where $[D]$ is the concentration of the drug. $I C_{50}$ represents the concentration of the blocker that causes $50 \%$ inhibition and $n$ is the Hill-coefficient.

\section{Voltage-dependent Behavior}

Voltage-dependence of activation was calculated in two steps: First, changes in driving force owing to the different test potentials were considered by calculating the conductance $g$ according to

$$
g=\frac{1}{V-E_{N a}}
$$

Thereafter, normalized data were fitted with a Boltzmann equation of the form:

$$
\frac{g}{g_{\max }}=\frac{1}{1+e\left(\frac{V_{50}-V}{k}\right)}
$$

Voltage-dependence of fast inactivation were fitted using a Boltzmann equation of the form:

$$
\frac{I}{I_{\max }}=\frac{1}{1+e\left(\frac{V-V_{50}}{k}\right)}
$$

In case of slow inactivation, Eq. 4 was extended by the additional parameter $(S)$ which considers the steady-state level of incomplete inactivation.

$$
\frac{I}{I_{\max }}=(1-S)\left(\frac{1}{1+e\left(\frac{V-V_{50}}{k}\right)}\right)+S
$$

Abbreviations used for voltage-dependent parameters: $V$ and $V_{50}$ are the actual clamp potential and the potential at which halfmaximal current $(I)$ or conductance $(g)$ occurs. $E_{\mathrm{Na}}$ indicates the reversal potential for sodium ions, which was experimentally determined for each cell. The slope factor is given by $k$.

\section{Interaction With the Inactivated State}

The interaction with the inactivated state cannot be directly analyzed, as this state is non-conducting. Therefore, different indirect approaches have been developed for this purpose.

1) In the first set of experiments, the drug-induced shift $\left(\Delta V_{50}\right)$ of the inactivation curves on the potential axis was used to estimate the affinity to the inactivated state $K_{i}$. For this 
purpose, the relative shift of the inactivation midpoints was plotted versus the concentration of TIC10. Data were fitted with the following equation (Bean et al., 1983):

$$
\Delta V_{50}=k * \ln \left(\frac{1+\frac{[D]}{K_{r}}}{1+\frac{[D]}{K_{i}}}\right)
$$

$K_{r}$ and $K_{i}$ denote the affinity for the resting and inactivated state, respectively. Other identifiers have the same meaning as before.

2) Estimation of apparent binding constants:

The affinity to the inactivated state $\left(K_{i}\right)$ was calculated here according to (Bean et al., 1983) as:

$$
\frac{1}{K_{a p p}}=\frac{h}{K_{r}}+\frac{(1-h)}{K_{i}}
$$

$K_{a p p}$ is the apparent affinity determined at a selected membrane potential at which the amount of non-inactivated channels (resting channels) is given by $h$ (determined from the preceding inactivation curve). $K_{r}$ is the affinity for the resting state, here $600 \mu \mathrm{M}$.

3) Time and concentration-dependent development of block:

The time constants $\left(\tau_{i}\right)$ of block development for different concentrations of TIC10 were estimated by double exponential fits of the form:

$$
\frac{I}{I_{\max }}=S+a_{1} * e^{\left(\frac{-t}{\tau_{1}}\right)}+a_{2} * e^{\left(\frac{-t}{\tau_{2}}\right)}
$$

$t$ denotes the time of inactivation, $\tau_{i}$ are individual time constants and $a_{i}$ represents the relative contribution of the two terms, with $S$ $+a_{1}+a_{2}=1$.

Association and dissociation rates:

Association $\left(k_{o n}\right.$ in $\left.\mu \mathrm{M}^{-1} \mathrm{~s}^{-1}\right)$ and dissociation rates $\left(k_{\text {off }}\right.$ in $\left.\mathrm{s}^{-1}\right)$ were estimated from the slope and the $y$-intercept of a linear regression where the inverse of the fast time constants $(1 / \tau)$ was plotted against the drug concentration [D] (Kuo et al., 1997).

$$
\frac{1}{\tau}=k_{o f f}+k_{o n} *[D]
$$

The corresponding dissociation constants were calculated according to:

$$
K_{d}=\frac{k_{o f f}}{k_{o n}}
$$

\section{Recovery From Inactivation}

Current amplitudes were normalized to the maximum amplitude of $I_{\mathrm{Na}}$ in the absence and presence of TIC10. Recovery time constants were estimated from double or triple exponential fits according to:

$$
\frac{I}{I_{\max }}=1-a_{1} * e^{\left(\frac{-t}{\tau_{1}}\right)}-a_{2} * e^{\left(\frac{-t}{\tau_{2}}\right)}-a_{3} * e^{\left(\frac{-t}{\tau_{3}}\right)}
$$

Variable identifiers have the same meaning as before, with $a_{1}+$ $a_{2}+a_{3}=1$.
All curve-fitting procedures were performed using SigmaPlot 13.0 (Sysstat, San Jose, California, United Stated).

\section{Nomenclature}

Upon activation of Nav1.5 channels the course of the current is commonly described by two phases for which different terms are in use. In the first phase the current rises to a maximal value from which it continuously declines upon channel inactivation. We use here the term "transient current" which is otherweise also called "early" or "peak current". The second phase is characterized by an almost very small current amplitude owing to the activity of non-inactivating channels. We use here the term "persistent current" which is identical to "late" or "plateau current".

\section{Statistics}

If not directly stated by the presence of error bars, graphs show representative data from single cells. Average values from at least $\mathrm{N}=5$ cells are given as mean $\pm \mathrm{SD}$ in the results section and in figure legends.

\section{RESULTS}

\section{TIC10 Blocks Human $\mathrm{Na}_{\mathrm{v}} 1.5$ Channels}

The principal capability of TIC10 angular (TIC10) to block VGSCs is shown in Figure 2. To this end, channel activations in the absence and presence of $10 \mu \mathrm{M}$ TIC10 were carried out by brief depolarizations to $-20 \mathrm{mV}$ using a holding potential of $-90 \mathrm{mV}$, which was close to half-maximal inactivation in this experiment. Under these conditions, the current amplitude obtained for control was reduced to about half of its size in the presence of $10 \mu \mathrm{M}$ TIC10 (Figure 2).

\section{Interaction With the Resting State}

As many sodium channel blockers exert their effects in a potential- or state-dependent manner, more specific investigations were undertaken, starting with the analysis for a probable interaction with the resting state. To this end, a double pulse protocol was conducted as illustrated in Figure 3B. Briefly, sodium channels were activated twice from a holding potential of $-140 \mathrm{mV}$. The first activation was performed in the absence of drug and served as control. The second activation was elicited after a $30 \mathrm{~s}$ preincubation of control or TIC10 containing solution. For evaluation, current amplitudes in the presence of TIC10 were related to their respective controls and plotted against the concentration of TIC10 (Figure 3A). Of note, the inhibitory effect was far from being half-maximal. Even at the highest concentration tested $(40 \mu \mathrm{M})$, the remaining current was still $93.2 \pm 4.3 \%$ that of control. Therefore, a calculated affinity $\left(\mathrm{K}_{\mathrm{r}}\right)$ can only be considered as a rough estimation for the interaction of TIC10 with the resting state. According to Eq. 1, the halfmaximal inhibition is calculated to occur at $600.4 \pm 290.8 \mu \mathrm{M}$ TIC10. Nevertheless, the inhibitory potency of TIC10 was strongly diminished when the channels were activated from a very negative holding potential $(-140 \mathrm{mV})$ compared to an activation from a membrane potential in the range of half- 

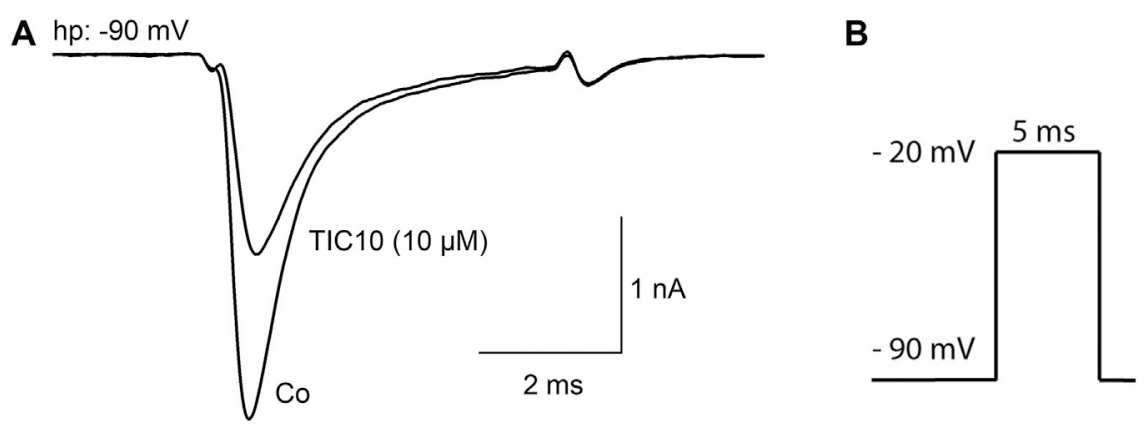

FIGURE 2 | Inhibition of $\mathrm{hNa} \mathrm{v}_{\mathrm{v}} 1.5$ channels by TIC10. (A) Representative current traces obtained for control and in the presence of $10 \mu \mathrm{M}$ TIC10. A similar outcome was obtained from four other cells. (B) Experimental scheme. Channel activations were carried out from a holding potential of $-90 \mathrm{mV}$ by depolarization to $-20 \mathrm{mV}$ for $5 \mathrm{~ms}$.

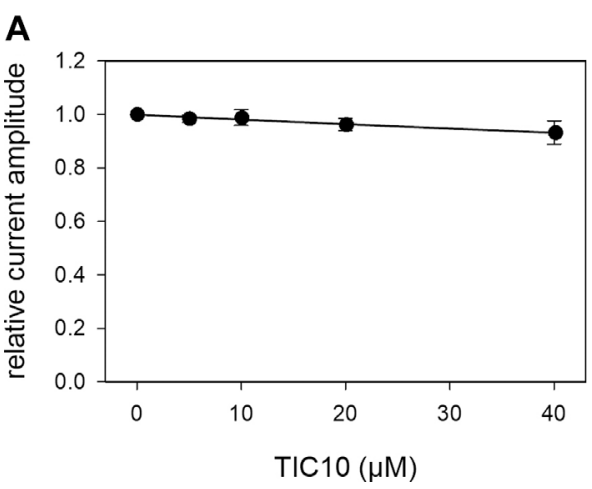

B

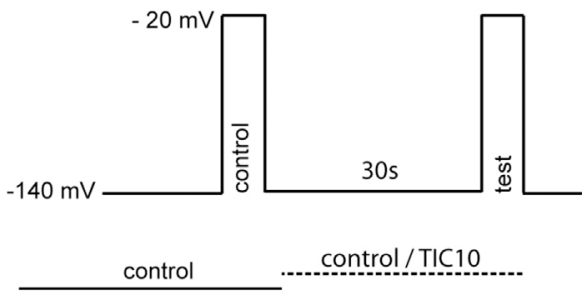

FIGURE 3 | Block of resting hNa 1.5 channels. (A) Illustrated are relative current amplitudes in relation to the applied concentration of TIC10. Data points were fitted according to Eq. 1. Note, even at the highest concentration of $\mathrm{TIC} 10(40 \mu \mathrm{M})$, the inhibitory effect is far from being half-maximal. Thus, the calculated affinity $\left(\mathrm{K}_{\mathrm{r}}=600.4 \pm\right.$ $290.8 \mu \mathrm{M}$ ) has to be considered as a rough estimation. (B) Experimental scheme. Channel activations in the absence (control) and presence (test) of different concentrations of TIC10 were carried out from a holding potential of $-140 \mathrm{mV}$. Current amplitudes obtained for the test-pulses were related to their controls and plotted against the concentration of TIC10.

maximal inactivation. Thus, it is obvious that the interaction of TIC10 strongly depends on the membrane potential.

\section{Voltage-Dependence of Activation}

A next set of experiments was conducted to determine if TIC10 interacts with the process of channel activation. Here, brief depolarizations from a holding potential of $-140 \mathrm{mV}$ to different test-pulse potentials (range: -90 to $+20 \mathrm{mV}$ in steps of $5 \mathrm{mV}$ ) were carried out (Figure 4B). Original traces thereof are illustrated in Figure 4A. From these traces, the maximal current amplitude of each sweep was plotted against the applied test-pulse potential, resulting in a biphasic curve (Figure 4C). For further evaluation, current amplitudes were converted to their corresponding conductances respecting the reversal potential (Eq. 2). Next, normalized conductances for control and in the presence of TIC10 were plotted versus the test-pulse potential, whereby activation curves were obtained (Figure 4D). The potential of half-maximal activation and the slope of the curve were obtained from a fit of data using Eq. 3. If the inhibitory effect of TIC10 is based on an interaction with channel activation, a shift of the activation curve to more positive potentials is expected. However, this does not apply here. By contrast, TIC10 even provoked a minor shift of the activation curve to more negative potentials. With full details, midpoints of activation curves averaged for control at $-43.1 \pm 2.9 \mathrm{mV}$ with a slope of $7.2 \pm$ $0.6 \mathrm{mV}$. The corresponding values in the presence of TIC10 $(10 \mu \mathrm{M})$ were $-44.8 \pm 2.8 \mathrm{mV}$ with a slope of $7.9 \pm 0.8 \mathrm{mV}$. Thus, the inhibitory impact of TIC10 on VGSCs does not arise from an interaction with the process of channel activation.

\section{Voltage-Dependence of Fast Inactivation}

The aim of the following experiment was to see if and how TIC10 interacts with the process of fast inactivation. To vary the amount of inactivated channels, pre-pulses of constant duration $(500 \mathrm{~ms})$ with an increasing pre-pulse potential (range -140 to $-45 \mathrm{mV}$ ) were applied immediately before the test-pulses (Figure 5B). Thereby, the number of inactivated channels increased as a function of the pre-pulse potential. For evaluation, all current amplitudes were related to the amplitude obtained from the most negative pre-pulse 

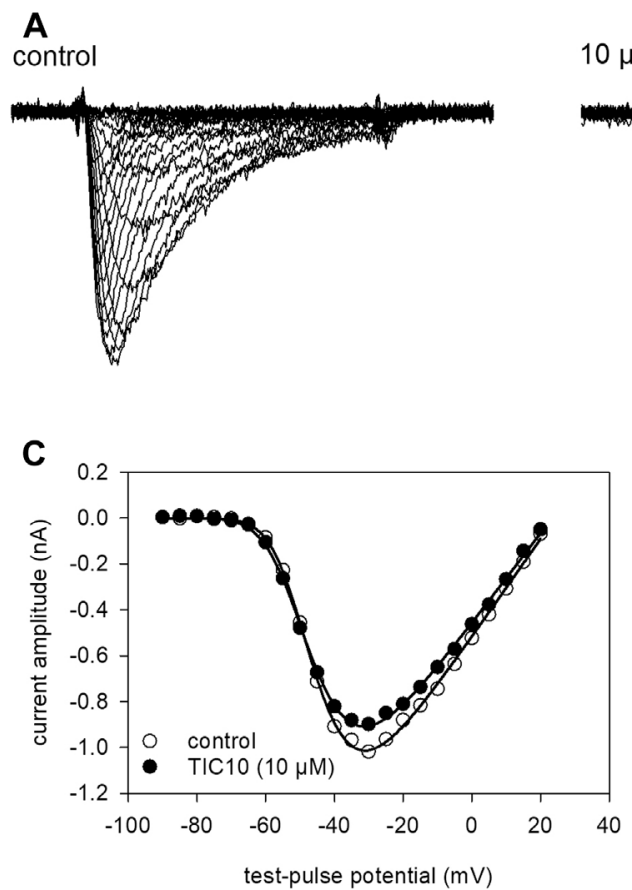

B

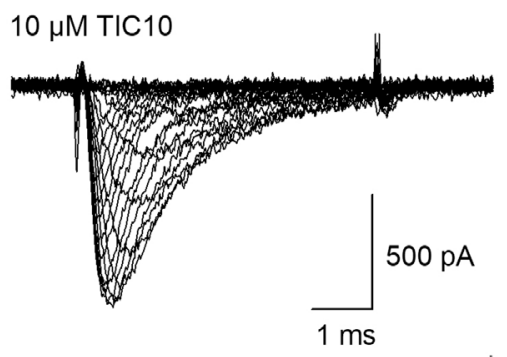

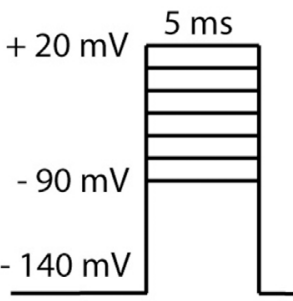

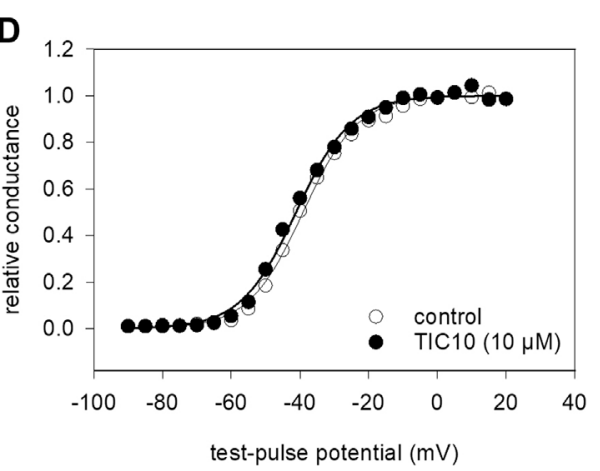

FIGURE 4 | Voltage-dependence of activation. (A) Original traces obtained from channel activations in the absence (left) and presence of $10 \mu \mathrm{M}$ TIC10 (right). (B) Experimental scheme: cells were clamped at a holding potential of $-140 \mathrm{mV}$ from which sodium currents were evoked by $5 \mathrm{~ms}$ test-pulses to potentials ranging from -90 to $+20 \mathrm{mV}$ (increment $5 \mathrm{mV}$ ). (C) Plots of maximal current amplitudes in the absence (open circles) and presence of $10 \mu \mathrm{M}$ TIC10 (filled circles) versus the test potential. (D) Voltage-dependence of channel activation. Data from C were converted to conductance, normalized, and plotted versus the test-pulse potential. Half-maximal activation occurred at $-43.1 \pm 2.9 \mathrm{mV}(\mathrm{k}: 7.2 \pm 0.6 \mathrm{mV}$ ) and $-44.8 \pm 2.8 \mathrm{mV}(\mathrm{k}: 7.9 \pm 0.8 \mathrm{mV})$ for control and in the presence of $10 \mu \mathrm{M}$ TIC10, respectively.

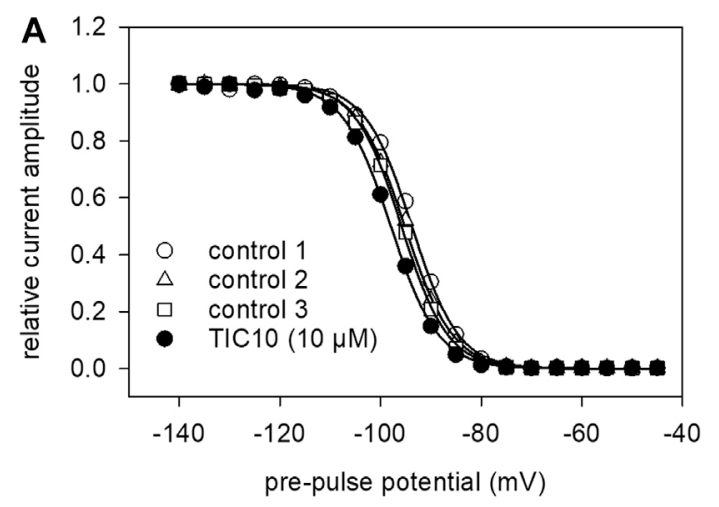

B

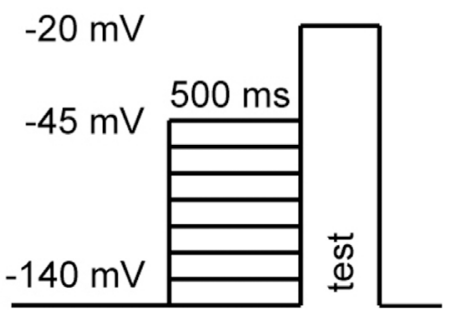

FIGURE 5 |Voltage-dependence of fast inactivation. (A) Data of normalized transient currents in the absence (open symbols) and presence of TIC10 (filled circles) are plotted against the pre-pulse potential. Solid lines represent fits according to Eq. 4. Respecting the drug independent shift, inactivation midpoints shifted in the presence of $10 \mu \mathrm{M} \mathrm{TIC10}$ by $1.2 \pm 1.4 \mathrm{mV}$ to more negative potentials. (B) Experimental scheme. Voltage-dependence of fast inactivation was determined by measuring sodium currents elicited by $5 \mathrm{~ms}$ depolarizations to $-20 \mathrm{mV}$ after conditioning pre-pulses for $500 \mathrm{~ms}$ to different potentials (range -140 to $-45 \mathrm{mV}$ ).

potential $(-140 \mathrm{mV})$. The relative current amplitudes were then plotted versus the pre-pulse potential. Data were fitted according to Eq. 4 in order to generate inactivation curves. The most important parameters here are the potential at which half of the channels are inactivated and the slope of the curve. If the inhibitory action of TIC10 is based on an interaction with fast inactivation a shift of the inactivation curve to more negative potentials is expected. As inactivation curves also undergo a drug independent left shift, mimicking an inhibitory effect, this shift was separately estimated in individual cells and taken into account in the evaluation of drug effects. The outcome of a typical experiment is illustrated 

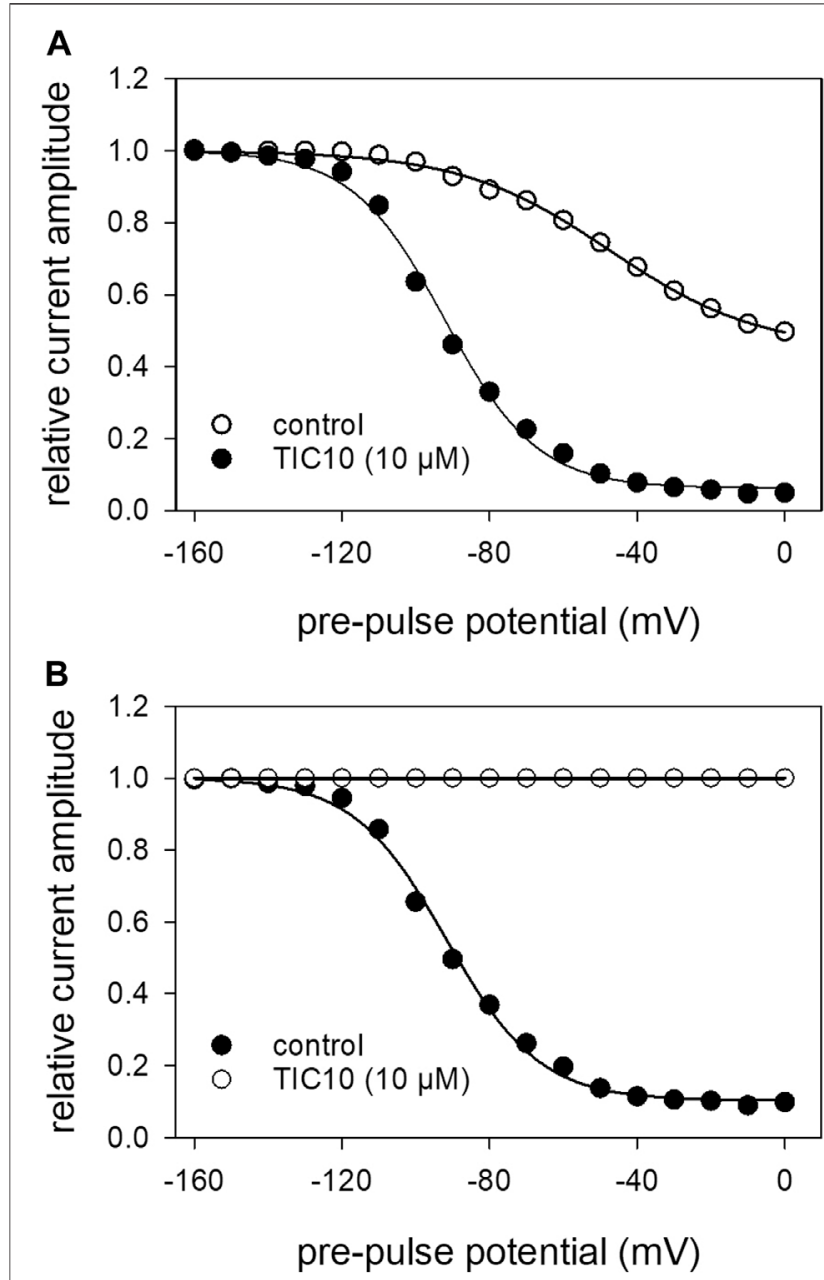

C

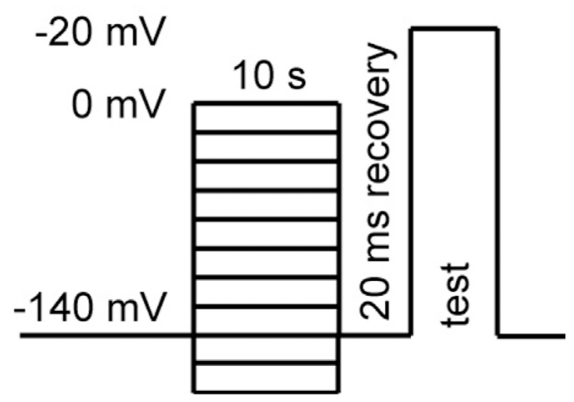

FIGURE 6 | Voltage-dependence of slow inactivation. (A) Relative current amplitudes in dependence of the pre-pulse potential. Solid lines are fits according to Eq. 5 for control (open circles) and TIC10 (10 $\mu \mathrm{M}$, filled circles). (B) Normalized data with resepct to control. (C) Experimental scheme. Activations to $-20 \mathrm{mV}$ were elicited after long-lasting (10 s) conditioning prepulses to different potentials (range: -160 to $0 \mathrm{mV}$; increment $10 \mathrm{mV}$ ) and a short (20 ms) recovery period at $-140 \mathrm{mV}$ immediately before the test-pulse.

by Figure 5A. Altogether, for $10 \mu \mathrm{M}$ TIC a shift of $-1.2 \pm$ $1.4 \mathrm{mV}$ was calculated. The slope was $5.3 \pm 0.9 \mathrm{mV}$ in the absence and $5.4 \pm 0.9 \mathrm{mV}$ in the presence of $10 \mu \mathrm{M}$ TIC10. These data indicate that TIC10 exerted no or only a minimal effect on fast inactivation.
Interaction With the Slow Inactivated State The protocol for analyzing an interaction with the slow inactivated state differed in two points from that of fast inactivation. First, the duration of the pre-pulse was prolonged to $10 \mathrm{~s}$. Second, immediately before the test-pulse a short recovery period $(20 \mathrm{~ms},-140 \mathrm{mV})$ was inserted to eliminate or minimize the contribution from fast inactivation (see Figure 6C). Under control conditions, the current amplitudes become smaller at prepulse potentials positive to around $-100 \mathrm{mV}$. Half-maximal inactivation occurred at $-42.9 \pm 5.0 \mathrm{mV}$ with a slope of $21.5 \pm$ $2.7 \mathrm{mV}$. At a pre-pulse potential of $0 \mathrm{mV}$, the remaining current amplitude amounted to $45.6 \pm 10.9 \%$. In the presence of TIC10, the slow inactivation curve was shifted to more negative values. In particular, half-maximal inactivation occurred at $-92.7 \pm 7.0 \mathrm{mV}$ with a slope of $12.8 \pm 0.9 \mathrm{mV}$. Furthermore, availability at $0 \mathrm{mV}$ dropped to $4.7 \pm 2.8 \%$ (Figure 6A). In order to ascribe this behavior either to an interaction with the fast or slow inactivated state, the potential-dependent behavior was inspected in more detail. As even under control, a clear potential dependency was obvious, TIC10-induced changes were normalized with respect to control by dividing drug values by control values (Figure 6B). If the inhibition is related to an interaction with the slow inactivated state, inhibition is expected to increase in the potential range where slow inactivation is likely to occur, i.e. in a potential range positive to that of fast inactivation. It turned out that the inhibition did not increase significantly in the range between -40 and $0 \mathrm{mV}$. In particular, inhibition amounted to $88.3 \pm 5.0 \%$ and $89.7 \pm 6.5 \%$, respectively. Taken together, these findings did not argue for an interaction with the slow inactivated state. Nevertheless, the interaction of TIC10 with the $\mathrm{hNa}_{\mathrm{v}} 1.5$ occurred on a slow time course.

\section{Time Dependence of Interaction}

Next, a set of experiments was performed to describe the time course of block development. The protocol is illustrated in Figure 7D. Briefly, after channel activation from a holding potential of $-140 \mathrm{mV}$ for control, ample time for full recovery at the holding potential was provided. Thereafter, the channels were inactivated for a variable duration (range: $25 \mathrm{~ms}-30 \mathrm{~s}$ ) at a potential of $-20 \mathrm{mV}$. Immediately before the test-pulse, channels were allowed to recover once more at the holding potential to eliminate or minimize the impact from fast inactivation. For evaluation, the current amplitudes obtained for test-pulses were related to their controls. Using this protocol in the absence of TIC10, relevant reductions of availability were observed at inactivation times lasting longer than $1 \mathrm{~s}$. The estimated time constant was $10.1 \pm 2.0 \mathrm{~s}$. At the longest inactivation time, the current amplitude dropped to $59.2 \pm 7.7 \%$. In the presence of TIC10, the availability already decreased at shorter inactivation times with a much more pronounced reduction at the longest inactivation time (Figure 7A). In particular, for $2.5 \mu \mathrm{M}$ TIC10 a time constant of $5.3 \pm 1.9 \mathrm{~s}$ with a remaining current of $46.0 \pm$ $4.8 \%$ was estimated. The corresponding values for $10 \mu \mathrm{M}$ TIC10 were $2.0 \pm 0.8 \mathrm{~s}$ and $16.9 \pm 6.4 \%$. For further evaluation, data were normalized with respect to control values (Figure 7B). To obtain time constants for block development, data were fitted with single 

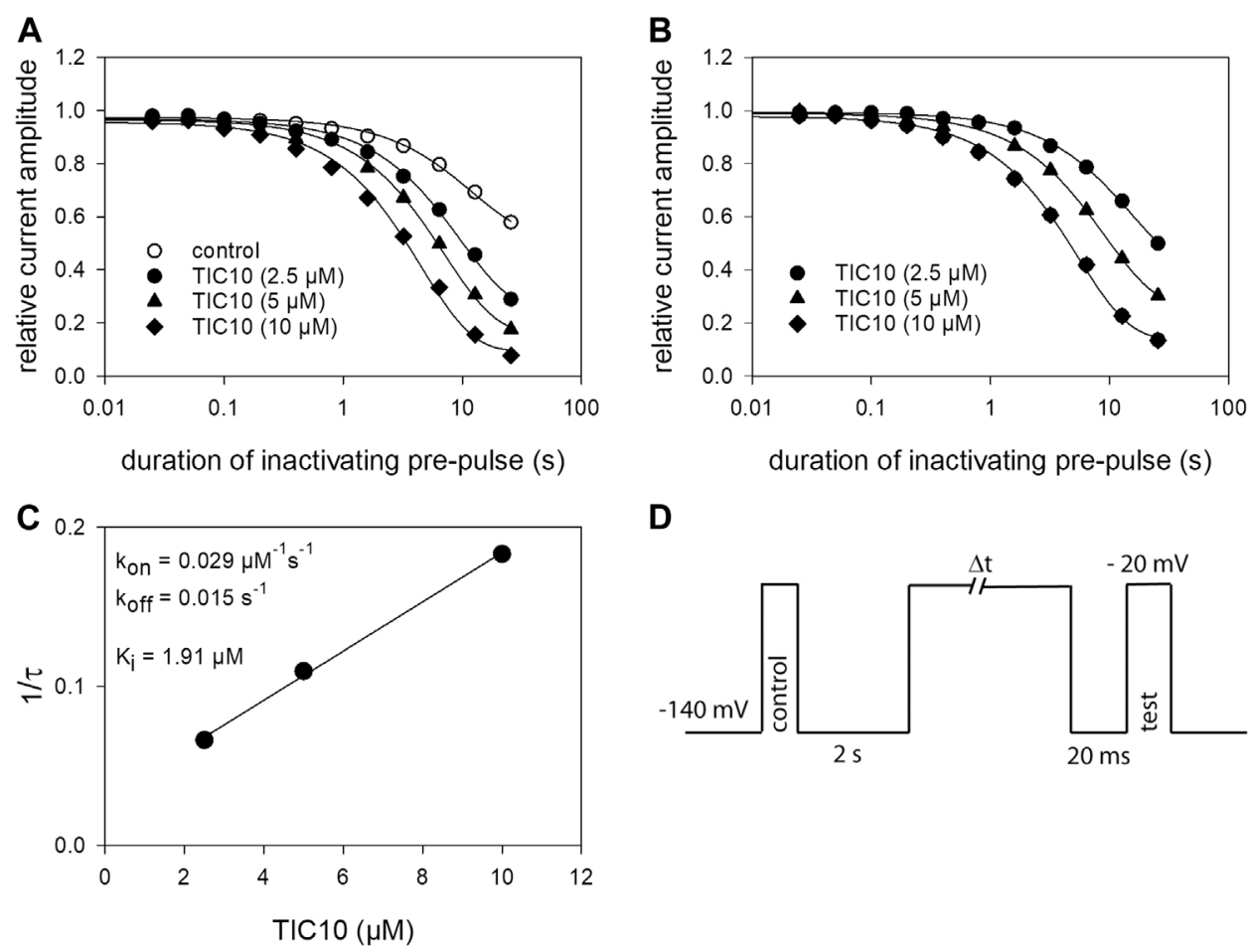

D

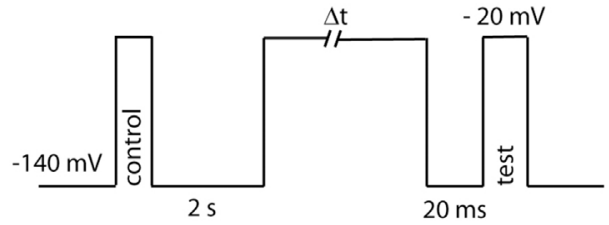

FIGURE 7 | Time-dependent interaction. (A) The graph illustrates relative current amplitudes in the absence and presence of TIC 10 versus the duration of an inactivating pre-pulse to $-20 \mathrm{mV}$. Solid lines describe individual time courses obtained in the absence and presence of TIC10 using single exponential functions for data fitting. (B) Data from A, normalized with respect to control. (C) Plot of the inverse of the time constants estimated from the data as illustrated in B versus the concentration of TIC10. $K_{o n}$ and $K_{\text {off }}$ values were taken from the slope and the $y$-intercept of the linear regression, respectively. The affinity for the inactivated state $\left(K_{i}\right)$ was calculated according to Eq. 10 to be $1.91 \mu \mathrm{M}$ in this experiment. Altogether a $\mathrm{K}_{i}$ of von $1.86 \pm 0.7 \mu \mathrm{M}$ resulted. (D) Experimental scheme. Starting from a holding potential of $-140 \mathrm{mV}$, first a control pulse to $-20 \mathrm{~s}$ was executed. Thereafter, ample time $(2 \mathrm{~s})$ was given to allow for full recovery before channels were inactivated for a variable duration at $-20 \mathrm{mV}$. The test-pulse was carried out after a short recovery at $-140 \mathrm{mV}$. For evaluation, test-pulse amplitudes were related to their controls.

exponential functions. Afterwards, the inverse, the inverse of the time constants was plotted versus the concentration of TIC10 (Figure 7C). The on-rate $\left(k_{\text {on }}\right)$ was directly taken from the slope of the linear regression and the off-rate $\left(k_{o f f}\right)$ was given by the $y$-intercept (Eq. 9, 10). For estimating the affinity for the inactivated state, the $K_{i}$ values were separately calculated for each cell according to the relation $K_{i}=k_{\text {off }} / k_{o n}$. The values for one representative cell are given as insert to Figure 7C. Overall, a $K_{i}$ of $1.86 \pm 0.7 \mu \mathrm{M}$ was determined.

\section{Interaction With the Inactivated State: Steady-State Parameters}

As TIC10 revealed strong effects after prolonged inactivation, additional experiments were conducted under these conditions to establish a full concentration-response relationship. To this end, a double pulse protocol was used as outlined by Figure 8B. Briefly, channel activations for control and in the presence of different concentrations of TIC10 were carried out after channels had been inactivated for $20 \mathrm{~s}$ at a potential of $-20 \mathrm{mV}$. To minimize drift effects during these long-lasting experiments, each run included its own control. Thus, drug effects as measured by the test-pulse were related to the timely executed control activation. Data analysis was restricted to cells where the current amplitude due to control- and test-pulse in the presence of mere control solution did not deviate by more than 3\%. Under these conditions, an affinity for the inactivated state with a $\mathrm{K}_{\mathrm{i}}$ of $2.0 \pm 0.5 \mu \mathrm{M}$ was calculated (Figure 8A). Interestingly, the $K_{i}$ values estimated from kinetic data (see previous section) and steady-state parameters are identical.

\section{Interaction With Partially Inactivated Channels}

In order to reflect more the physiological situation, concentrationrelationships were performed at a presumed resting membrane potential of $-90 \mathrm{mV}$ were the channels exist in the fast and slow as well as in the resting state. Again, the experimental scheme consisted of a double pulse protocol that deviated from the previous one by the potential for inactivation and the lack of a recovery period before the control- or test-pulse (Figure 9B). In any case, the inactivation potential was estimated for each cell individually from another double pulse protocol, which was restricted to the relevant part of the inactivation curve. For evaluation, the current amplitudes obtained for the test-pulses were related to their controls and plotted versus the concentration of TIC10 (Figure 9A). Data points were fitted with Eq. 1, whereby so-called apparent affinity constants $\left(\mathrm{K}_{\mathrm{app}}\right)$ 


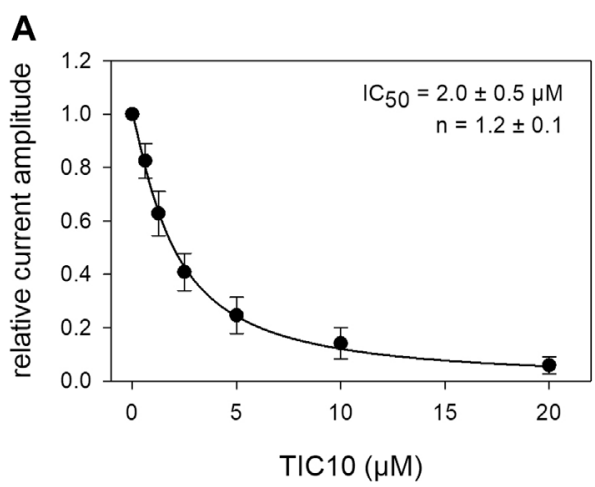

B

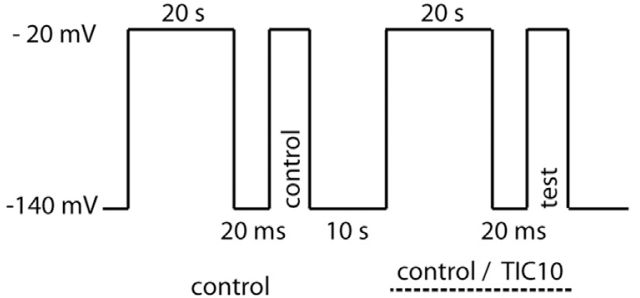
presence of different concentrations of TIC10, after a prolonged inactivation. (B) Experimental scheme, illustrating the double pulse protocol. Starting from a holding potential of $-140 \mathrm{mV}$ channels were inactivated for $20 \mathrm{~s}$ at a potential of $-20 \mathrm{mV}$. After a short recovery at holding potential, the control pulse was executed. Between the control and test run (second part), ample time (10 s) for full recovery was provided. In the second part, the protocol was repeated in either control or test solution. For evaluation, test current amplitudes were related to their controls. Data analysis was restricted to data sets where control and test-pulse amplitude in mere control solution did not deviate by more than $3 \%$. Overall, half-maximal inhibition $\left(\mathrm{IC}_{50}\right)$, representing the affinity for the inactivated state $\left(\mathrm{K}_{\mathrm{i}}\right)$ was calculated to be $2.0 \pm 0.5 \mu \mathrm{M}$.

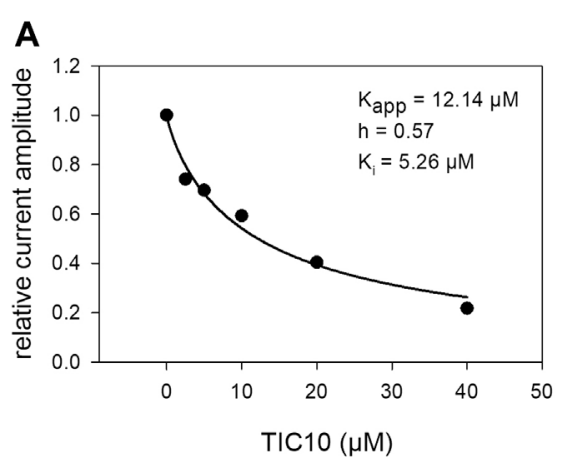

B

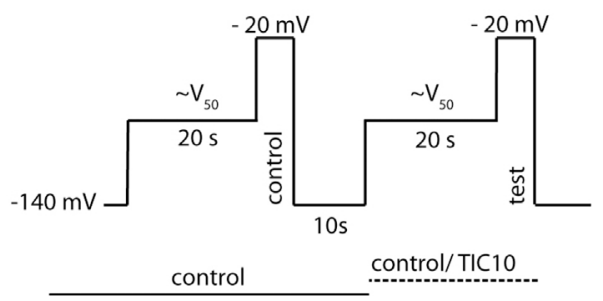

FIGURE 9| Interaction with partially inactivated channels. (A) Illustrated are relative currents amplitudes in relation to the applied concentration of TIC10 upon partial inactivation of the channels of one representative cell (out of five experiments). For evaluation data were fitted with Eq. 1, giving $K_{\text {app. }}$ In order to estimate the affinity for the inactivated state $\left(\mathrm{K}_{\mathrm{i}}\right)$, the current amount of non-inactivated channels (h) was considered according to Eq. 7, resulting in a mean $\mathrm{K}_{i}$ of $4.7 \pm 1.3 \mu \mathrm{M}(\mathrm{N}=5)$. Data evaluation (relation of test-pulse to control pulse) was restricted to experiments where the current amplitudes obtained from mere control solution did not deviate by more than $3 \%$. (B) Experimental scheme. From a holding potential of $-140 \mathrm{mV}$ conditioning pre-pulses to a potential of about half-maximal inactivation $\left(\mathrm{V}_{50}\right)$ were applied for $20 \mathrm{~s}$ before the test-pulse to $-20 \mathrm{mV}$ was executed. This protocol was executed a second time, either in the presence of control solution or different concentrations of TIC10.

resulted. The word "apparent" is used to indicate that the resulting affinity constant is composed from the affinity to the resting and the affinity to the inactivated state. In order to recalculate the affinity for the inactivated state, Eq. 7 was used. This requires the affinity for the resting state $(600 \mu \mathrm{M})$, obtained from previous experiments, and the current amount of inactivated channels, which was estimated in individual cells immediately before starting the experiments. As the current amount of inactivated channels varies between individual cells, each cell was evaluated separately. Altogether, an affinity to the inactivated state of $4.7 \pm 1.3 \mu \mathrm{M}$ resulted. Of note, the affinity for the inactivated state estimated here is less than that obtained from the other experiments.

\section{Recovery From Inactivation}

If a drug interacts with the inactivated state (fast or slow), it is expected that also recovery from inactivation is affected. We analyzed this aspect by the use of a tri-pulse experiment
(Figure 10B). After an initial control pulse, sufficient time was provided for full recovery. Next channels were inactivated for $500 \mathrm{~ms}$ at $-20 \mathrm{mV}$. The test-pulse was initiated after channels had been allowed to recover at a potential of $-140 \mathrm{mV}$ for a variable time (range: $0.2 \mathrm{~ms}$ to $2.7 \mathrm{~s}$ ). For evaluation, the current amplitudes of the test-pulses were related to their controls and plotted versus the recovery time (Figure 10A). To describe the time course of recovery, data points were fitted with double exponential functions (Eq. 11). Under control condition, most of the channels $(92.6 \pm 4.0 \%)$ recovered with a time constant of $\tau_{1}=1.4 \pm 0.6 \mathrm{~ms}$, the remainder with $\tau_{2}=23.6 \pm 12.0 \mathrm{~ms}$. In the presence of $10 \mu \mathrm{M}$ TIC10, the proportion of fast recovering channels decreased to $75.6 \pm 7.0 \%$ while the recovery time slightly increased to $2.3 \pm 1.2 \mathrm{~ms}$. The most prominent effect of TIC10 here was the about tenfold increase of the second time constant to $267.6 \pm 73.1 \mathrm{~ms}$. In order to estimate recovery from slow inactivation, the experimental scheme was modified to a 

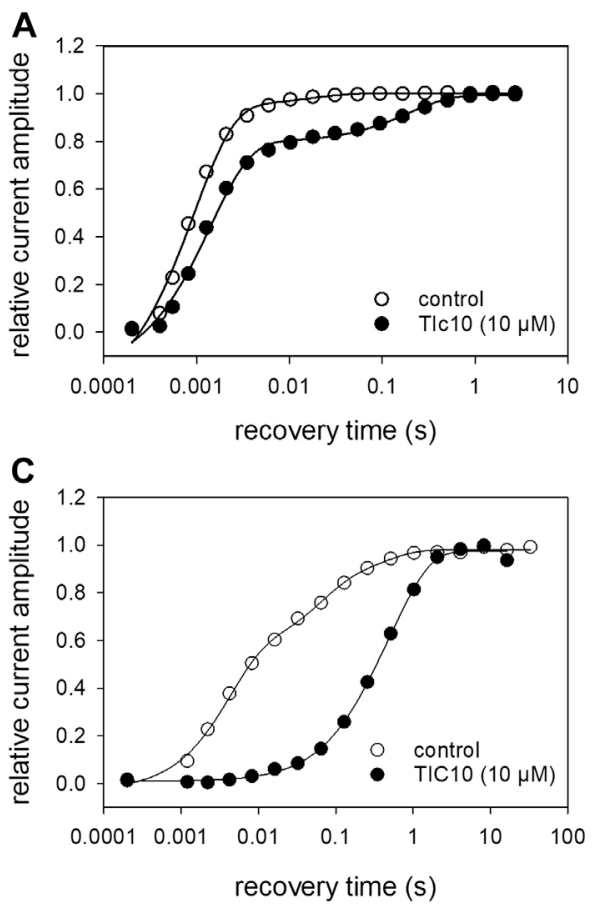

B

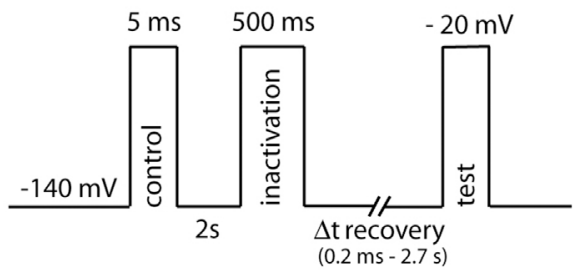

D

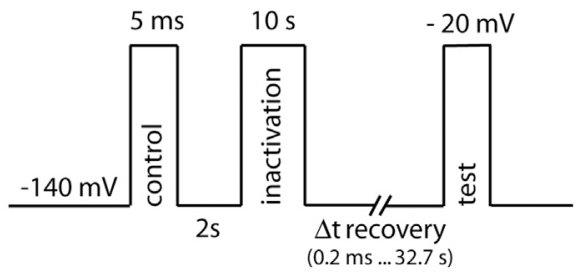

FIGURE 10 | Recovery from inactivation. (A) Recovery from fast inactivation. The relative amount of available channels versus the recovery time at -140 mV is illustrated. Solid lines represent fits with two exponential functions according to Eq. 11. (B) Experimental scheme. After an initial control pulse, inactivation was performed at $-20 \mathrm{mV}$ for $500 \mathrm{~ms}$. The test-pulse was applied after a varying time of recovery. For evaluation, current amplitudes of the test-pulses were related to their controls. Interval between individual runs was $10 \mathrm{~s}$ and TIC10 was applied at $10 \mu \mathrm{M}$. (C) Recovery from slow inactivation. In case of control, three exponentials were required to describe the time course of recovery; in the presence of TIC10, two exponentials were sufficient. (D) Experimental scheme for recovery from slow inactivation. Otherwise, identical protocol as in (A) but with the inactivation time set to $10 \mathrm{~s}$ and recovery time prolonged up to $32.8 \mathrm{~s}$.

TABLE 1 | Time constants for recovery from slow-inactivation of TIC10 (10 $\mu \mathrm{M})$.

\begin{tabular}{lcccc} 
& Control & Amount & TIC10 & Amount \\
\hline$\tau_{1}(\mathrm{~ms})$ & $4.8 \pm 4.9$ & $53.3 \pm 5.2 \%$ & $128.1 \pm 80.3$ & $36.3 \pm 16.0 \%$ \\
$\tau_{2}(\mathrm{~ms})$ & $54.4 \pm 45.2$ & $31.0 \pm 4.5 \%$ & $548.4 \pm 287.4$ & $62.2 \pm 15.7 \%$ \\
$\tau_{3}(\mathrm{~ms})$ & $486.9 \pm 389.0$ & $14.8 \pm 2.9 \%$ & - & -
\end{tabular}

prolonged inactivation time (10 s) and an expansion of the recovery time up to $32.8 \mathrm{~s}$ (Figure 10D). As expected, the effect of TIC10 was more pronounced here (Figure 10C). Under control, three exponentials were required to describe the time course of recovery, whereas in case of TIC10, two time constants were sufficient (Table 1).

\section{Tests for an Open Channel Blocking Mechanism-Interaction With the Persistent Current.}

There are several protocols in use to test for an open channel blocking mechanism. In this work, two of them were used: Use-dependency and inactivation-deficient mutants. In a first set of experiments, we looked for a use-dependent behavior. To this end, short test-pulses (1 ms) of $-20 \mathrm{mV}$ were applied from a holding potential of $-140 \mathrm{mV}$ (Figure 11B). We fixed on this short activation time in order to minimize the inactivation time during each activation cycle. Prior to the high frequency stimulation, infrequent activations were carried out $(0.2 \mathrm{~Hz})$ until a stable baseline was achieved. Thereafter, either control solution or TIC10 was perfused for $20 \mathrm{~s}$ before the high frequency stimulation $(10 \mathrm{~Hz})$ was started. For evaluation, the current amplitude of each pulse was divided by the amplitude of the first response of the high frequency stimulation train. For control, current amplitude minimally declined to $99.5 \pm 0.6 \%$. In the presence of TIC10, the remaining current of the last activation amounted to $95.7 \pm 1.6 \%$ (Figure 11A). Thus, TIC10 revealed no or only a minimal use-dependent behavior.

In the next experiments, we tested a channel mutant with reduced capability to inactivate $\left(\mathrm{hNa}_{\mathrm{v}} 1.5 \_\mathrm{I} 408 \mathrm{~W} \_\mathrm{L} 409 \mathrm{C}\right.$ A410W; WCW mutant). With this mutant, the channels stay open to a large extent even after a prolonged activation $(500 \mathrm{~ms})$, as evidenced by a persistent current. If a drug leads to a concentration-dependent reduction of this persistent current, it can also be regarded as an interaction with the open state. Original traces from these experiments are illustrated in Figure 12A. It is evident that the tranisent current, representing drug binding to the resting state, is hardly affected in the presence of TIC10 up to a concentration of $40 \mu \mathrm{M}$ (Figure 12B). Thus, no estimation of the half-maximal effective concentration was performed. By contrast, the persistent current was strongly diminished in a concentrationdependent manner with a half-maximal inhibition occurring at $4.1 \pm 1.4 \mu \mathrm{M}$ TIC10 with a Hill-coefficient of $0.9 \pm 0.1$. Thus, TIC10 also operated as an open channel blocker. 


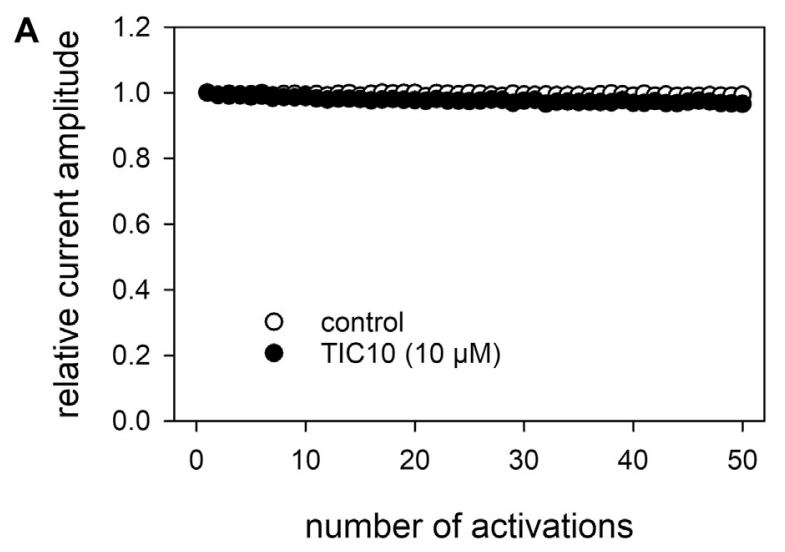

B

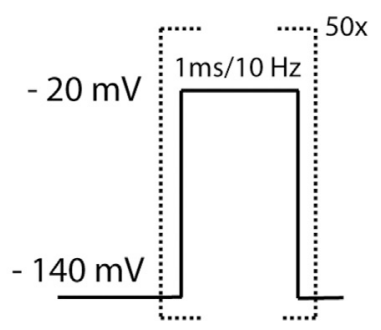

FIGURE 11 | Analysis for use-dependence. (A) Illustrated is the relative current amplitude versus the number of channel activations. (B) Experimental scheme. Channel activations were carried out by brief pulses $(1 \mathrm{~ms})$ to $-20 \mathrm{mV}$ at a frequency of $10 \mathrm{~Hz}$ in the absence and presence of TIC10 (10 $\mu \mathrm{M})$. Current amplitudes minimally decline under control from the first to the last activation (50th pulse) to $99.5 \pm 0.6 \%$ and in the presence of $\mathrm{TIC} 10$ (10 $\mu \mathrm{M})$ to $95.7 \pm 1.6 \%$. TIC10 was preincubated for $20 \mathrm{~s}$ before starting the high frequency activation.

\section{Identification of the Possible Interaction Site by Channel Mutants}

To look for a possible interaction site at the $\mathrm{hNa}_{\mathrm{v}} 1.5$, we used two mutants, F1760K and N406C. The F1760K mutant is one out of several mutants, which affects the local anesthetic binding site (Catterall, 2014). This site is not only important for local anesthetics but also for many blockers of VGSCs. If a drug primarily interacts at the position 1760, a strongly reduced affinity is expected in case of the F1760K mutant. This clearly applied for TIC10. The estimated affinity for the slow inactivated channels was $46.2 \pm 16.9 \mu \mathrm{M}$ (Hill-coefficient: $1.5 \pm$ 0.1 ), which is more than 20 -fold less affine compared to the wild type (Figure 13A). Another important interaction site which affects the affinity for local anesthetics is N406. This site additionally has an impact on slow inactivation (McNulty et al., 2006). Using the N406C mutant, the affinity for TIC10 marginally changed compared to the wild type (Figure 13B). In particular, the estimated $\mathrm{IC}_{50}$ was $3.3 \pm 0.6 \mu \mathrm{M}$ with a Hillcoefficient of $1.2 \pm 0.1$.

\section{Investigation of Imipridone Derivatives}

Beside TIC10 angular, two other imipridones were of special interest. These were the linear isoform of TIC10 (TIC10 $0_{\text {linear }}$ ) and a fluorinated derivative, called ONC212 (Figure 1). Concerning the anti-tumoral activity, $\mathrm{TIC}_{10} 0_{\text {linear }}$ is nearly inactive, while ONC212 is more than 10 times more effective than TIC10 angular (Lev et al., 2017; Wagner et al., 2017; Nii et al., 2019). With respect to our investigations, we were interested to investigate if this anti-tumoral behavior is paralleled in the interaction with the $\mathrm{hNa}_{\mathrm{v}} 1.5$. For this purpose, only selected protocols were used. The experimental data show that in case of ONC212 the affinity for the slow inactivated state (according to Figure 8B) is nearly identical to that of TIC10 angular while $\mathrm{TIC}_{10} 0_{\text {linear }}$ was about five-times less effective (Figure 14). In particular, the affinity for ONC212 was $2.5 \pm 0.7 \mu \mathrm{M}$ (Hillcoefficient $1.5 \pm 0.1$ ) and that for TIC10 $0_{\text {linear }}$ was $11.2 \pm 1.3 \mu \mathrm{M}$
(Hill-coefficient $1.0 \pm 0.1$ ). In contrast to TIC10 ${ }_{\text {angular, }}$ the time course for block development had to be described for both compounds with two time constants (Figure 15). Data, listing the time constants and the relative amounts of the individual terms are summarized by Table 2 . Due to the additional fast component, it was obvious that the derivatives might additionally affect fast inactivation. Indeed, this was the case for both compounds with a stronger effect obtained for ONC212 (Figure 16). ONC212 $(10 \mu \mathrm{M})$ shifted mid points of fast inactivation by $-4.7 \pm$ $0.4 \mathrm{mV}$ and $\mathrm{TIC} 10_{\text {linear }}(10 \mu \mathrm{M})$ by $-3.0 \pm 0.4 \mathrm{mV}$. The possible interaction with the open state was analyzed using the WCW mutant. Both derivatives revealed here affinities close to $\mathrm{TIC}_{10}{ }_{\text {angular }}$ (Figure 17). In particular, for $10 \mu \mathrm{M}$ ONC212, the calculated value was $4.8 \pm 0.5 \mu \mathrm{M}$ (Hill-coefficient: $1.4 \pm 0.2$ ); the corresponding value for $10 \mu \mathrm{M}$ TIC10 1 linear was $3.5 \pm 0.7 \mu \mathrm{M}$ (Hillcoefficient $1.1 \pm 0.2$ ). Altogether, it turned out, that the anti-tumor potency of the analyzed compounds was only partly mirrored in individual experimental designs used to analyze their interaction with the $\mathrm{hNa}_{\mathrm{v}} 1.5$.

\section{DISCUSSION}

TIC10, later renamed to ONC201, belongs to a novel class of anticancer compounds called imipridones (Allen et al., 2016; Anderson and Scott, 2017). Among this group, several derivatives exist with a varying efficacy concerning anti-tumor activity (Wagner et al., 2017). There are several mechanisms of action that were reported to contribute to the anti-tumor activity of TIC10. First, the induction of TRAIL (Allen et al., 2013). Second, an interference with the dopamine receptor $\mathrm{D}_{2}$ (Kline et al., 2018). Third, inhibition of oxidative phosphorylation by hyperactivation of the mitochondrial caseinolytic protease $\mathrm{P}$ (ClpP) (Ishizawa et al., 2019). Here, we described an as far unknown pharmacological property of TIC10, which might contribute to its anti-tumor activity: TIC10 is a potent blocker of VGSCs. We determined that it interacts with the $\mathrm{hNa}_{\mathrm{v}} 1.5$ in a 


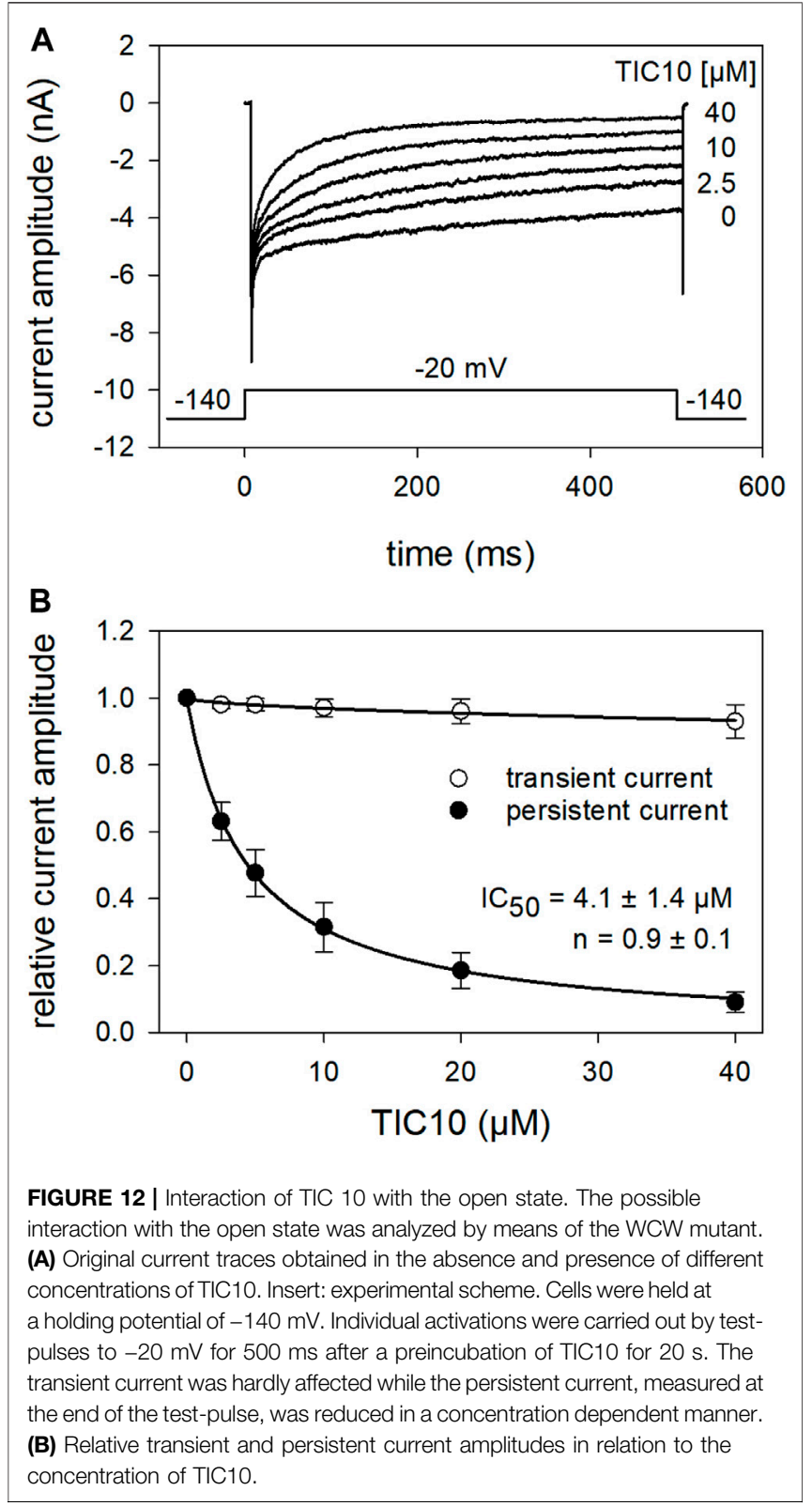

state- but not use-dependent manner. The interaction of TIC10 with different channel states occurs well within the concentration range in which it is used in tumor therapy.

\section{Voltage-Gated Sodium Channels and Cancer}

The functional expression of VGSCs in tumor cells has initially been described for prostatic cancer cells (Grimes et al., 1995). Meanwhile, corresponding observations have been made for many other cancer cells. Altogether, there is no doubt that voltage-gated sodium channels play an important role in metastasis, especially in the processes controlling migration and invasion of tumor cells. Accordingly, blockade of VGSCs or reducing their expression level is expected to have beneficial

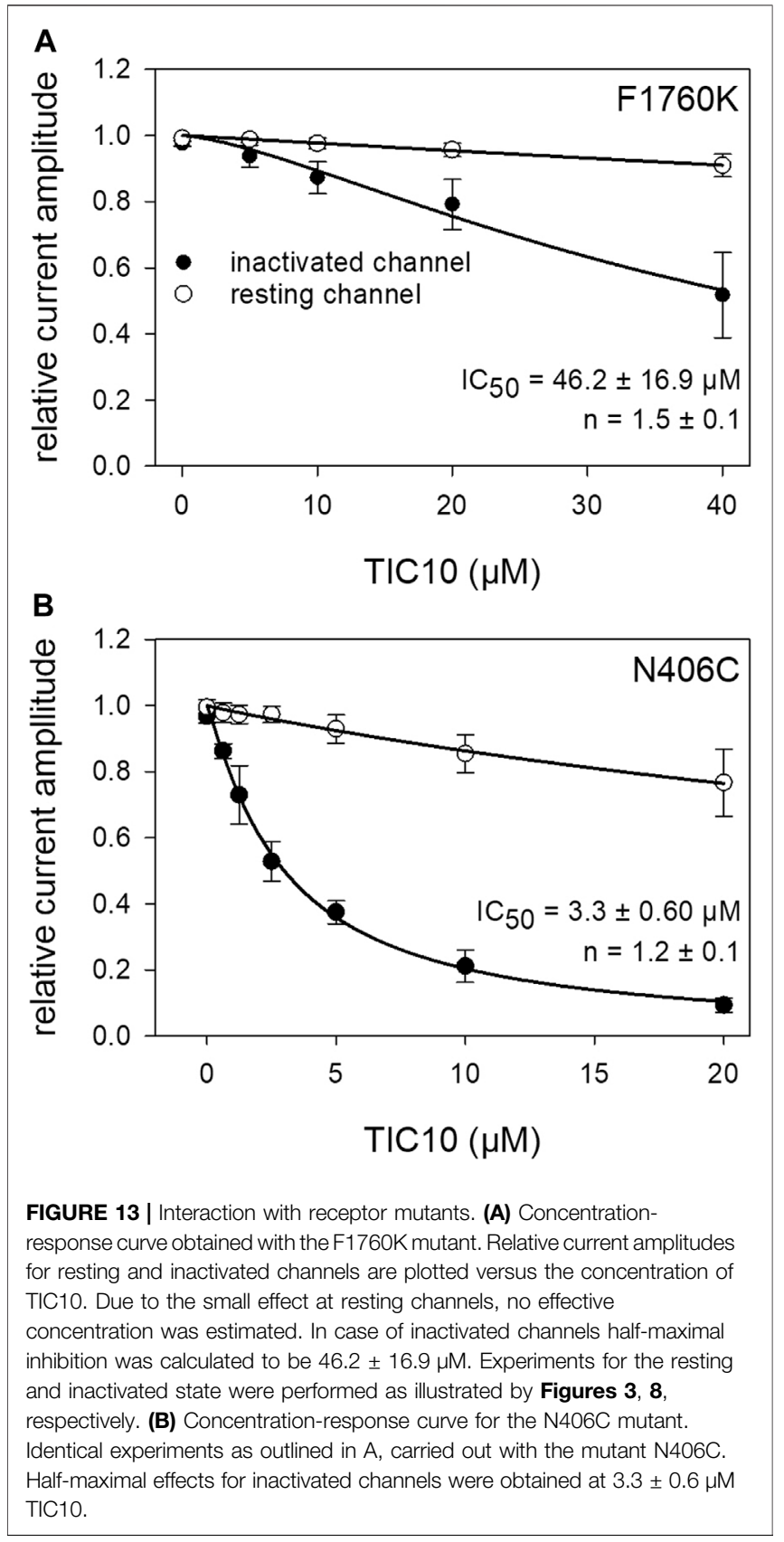

effects in tumor therapy (Djamgoz and Onkal, 2013; Djamgoz et al., 2019).

In the past, the occurrence of VGSCs has been associated with excitable cells where they are required for generating action potentials. Meanwhile, mRNA encoding for this type of channel has been detected in a broad variety of non-excitable cells, including cancer cells. In the latter, mRNA levels of VGSCs can be upregulated more than 1000-fold, with the amount of VGSCs directly correlating to their metastatic potency (Fraser et al., 2005). Of note, the functional expression of VGSCs in tumor cells is much less than mRNA, but even strong enough to make these cells potentially excitable. However, excitability does 
not seem to be of relevance VGSCs play in tumorigenesis (Djamgoz et al., 2019). Moreover, it is their capability to be selectively permeable for sodium ions, whereby an increased level of the concentration of intracellular sodium ions results. Indeed, an elevated concentration of sodium ions in the tissue and in the cytosol of malignant cells has been found (Ouwerkerk et al., 2007; Li et al., 2015). It is evident that increased intracellular sodium levels cannot be achieved by the minute amount of sodium influx, which would result from brief channel openings (Roger et al., 2004; Carter and Bean, 2009). Aside from this, such activations are very unlikely to happen regarding the rather positive resting membrane potential (range: -40 to $-20 \mathrm{mV}$ ) of these cells, which makes the VGSCs not available for activation (Roger et al., 2015). Once more, the relative positivity of the resting membrane potential of the analyzed cell lines correlates with their metastatic potency (Fraser et al., 2005). Thus, two ways for a permanent sodium influx are in focus: first, a window current in the range from -60 to $-20 \mathrm{mV}$ resulting from an incomplete overlap of the VGSCs activation and inactivation curves, with the maximum current at the more negative end of the resting potential range observed in tumor cells (Djamgoz and Onkal, 2013). Second, a persistent, non-inactivating sodium current (INaP) the activity of which is not restricted to a narrow potential range as it is also observed at depolarized membrane potentials (Maltsev et al., 1998). This latter current is also enhanced under hypoxia, a condition commonly observed in the tumor environment (Ju et al., 1996; Brahimi-Horn and Pouysségur, 2007). All in all, this persistent sodium current is favored as the base for the increased intracellular concentration of sodium ions which in turn activates the sodium-hydrogen exchanger 1 (NHE1). By the export of protons, an extracellular acidification is achieved, which is a prerequisite for proteolytic enzymatic activity providing the basis for invasiveness (Djamgoz et al., 2019). A pharmacological hallmark of the INaP is, that it can be blocked by almost all blockers of VGSCs as this current flows through the same channels as the transient, fast inactivating current (INaT) (Urbani and Belluzzi, 2000). Furthermore, there are also drugs (mexiletine, flecainide, ranolazine) which preferentially interact with this state while being less effective in other settings (Wang et al., 2003, 2004, 2008). However, a mere selectively targeting of the $\mathrm{INaP}$ is not a sufficient criterion for choosing and applying a sodium channel blocker as the different interactions of a particular blocker must be evaluated in toto.

\section{Impact of $\mathrm{TIC}_{10}$ angular}

TIC10 blocked the $\mathrm{hNa}_{\mathrm{v}} 1.5$ in a state-dependent manner. The interaction with the resting state was very low (around $600 \mu \mathrm{M}$ ), while the affinity to the inactivated state was about $2 \mu \mathrm{M}$. The ratio in affinity for the resting versus the inactivated state is generally regarded as a safety margin, the higher the better (Ilyin et al., 2005). To be judged as safe, the ratio should be at least 50fold. In case of TIC10, the ratio is about 300 -fold, which rates TIC10 as a safe drug in this setting. Next, the interaction of TIC10 with the $\mathrm{hNa}_{\mathrm{v}} 1.5$ happens in a slow manner whereby an interaction with INaT is rather unlikely. This applies to a single activation as well as for repeated activations as observed in the test analyzing for a use-dependent behavior. Concerning heart activity, this is a favorable property, as affecting INaT would potentially be proarrhythmic due to an intracardial conduction delay. Indeed, a prolongation of the QRS complex can be observed by current reductions as little as $10 \%$ (Poulin et al., 2014). In this way, it has been proposed earlier that an ideal VGSC blocker should only block the persistent current (INaP) while leaving the transient current (INaT) unaffected (Noble, 2006). The main problem for the analysis of drug effects on the INaP consists of its small size, which in wild-type channels is almost less than $1 \%$ compared to the corresponding INaT. Therefore, different experimental approaches have been developed in order to amplify its size. These include enzymatic or chemical treatment, the application of toxins, or the use of inactivation-deficient mutants (Grant et al., 2000; Belardinelli et al., 2013; Wang et al., 2013). However, it should be kept in mind, that all these manipulations might also have unspecific effects on drug interaction (Föhr et al., 2021). We performed our experiments with an inactivation-deficient mutant. Again, INaT was hardly affected while INaP revealed an affinity of about $4 \mu \mathrm{M}$, which is well beyond the maximal concentration found in the plasma of treated patients (Allen et al., 2016). In further experiments, we obtained hints that the interaction of TIC10 with the $\mathrm{hNa}_{\mathrm{v}} 1.5$ most probably happens via the binding site for local anesthetics as the affinity of the corresponding mutant was more than 20 -fold lower as for wildtype channels. Thus, TIC10 uses the same mechanism/ interaction site as the majority of blockers of VGSCs.

These findings make it unlikely that TIC10 can also discriminate between the adult and neonatal form of the $\mathrm{hNa}_{\mathrm{v}} 1.5$. Of course, a drug which blocks the neonatal form while leaving the adult form unaffected would be highly desired (Roger et al., 2004; Fraser et al., 2005). An important point here is that tumor cells, which express $\mathrm{hNa}_{\mathrm{v}} 1.5$ channels, preferentially express the neonatal form, whereas the neonatal form is expected to be absent to a large extent from normal adult tissue (Onkal and Djamgoz, 2009; Yamaci et al., 2017). So far, to the best of our knowledge, excepting a polyclonal antibody, no drug is known to discriminate between the adult and neonatal form of the $\mathrm{hNa}_{\mathrm{v}} 1.5$, including our previous study about the inhibitory effect of IC261 on these channels (Föhr et al., 2017). The main point here is that the two forms differ by only seven amino acids in segment three and four of domain I introducing one additional positive charge whereby amongst others channel activation will be affected (Walzik et al., 2011). However, as blockers of VGSCs, which affect channel activation, are extremely rare, it remains challenging to discover or design corresponding drugs.

Furthermore, it is tentative to speculate that the inhibitory action of TIC10 might not be restricted to the $\mathrm{hNa}_{\mathrm{v}} 1.5$ as statedependent blocker often reveal a low isotype specificity (Ilyin et al., 2005). This might be of importance as almost all isoforms have been reported to occur in different tumors, whereby the isoform itself seems not to be critical for the biological effect in cancer (Roger et al., 2015). Furthermore, all VGSCs expressed in cancer cells also reveal an INaP irrespective of the subtype, at least under hypoxic conditions (Djamgoz et al., 2019). 

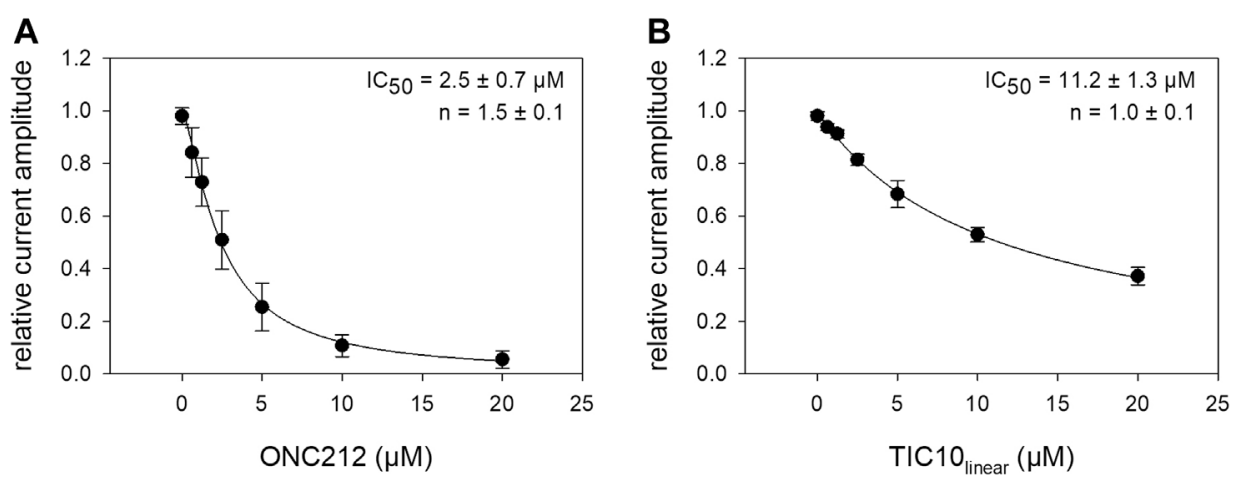

FIGURE 14 | Interaction of imipridone derivatives with the inactivated state. The graphs show relative current amplitudes upon channel activations in the presence of different concentrations of imipridone derivates after a prolonged inactivation (20 s). Experimental scheme as illustrated by Figure 8B. (A). Data for ONC212, revealed a half-maximal inhibition at $2.5 \pm 0.7 \mu \mathrm{M}$. (B) Data for TIC10 linear with a half-maximal effect at $11.2 \pm 1.3 \mu \mathrm{M}$.
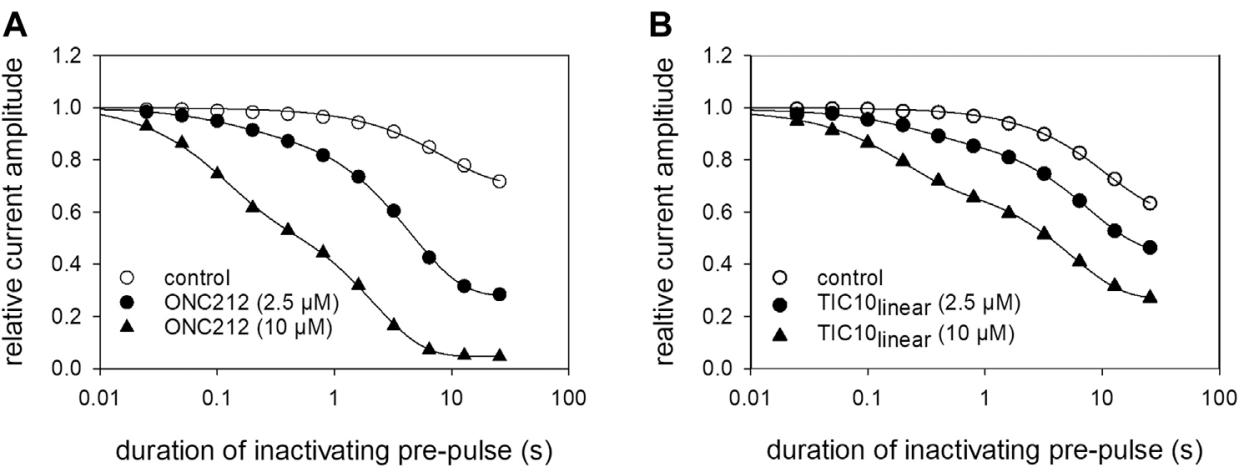

FIGURE 15 | Time-dependent interaction of imipridone derivatives. The graph illustrates relative current amplitudes in the absence and presence of different concentrations of (A) ONC212 and (B) TIC10 linear versus the duration of the inactivating pre-pulse to $-20 \mathrm{mV}$. Solid lines describe individual time courses obtained in the absence and presence of TIC10 using double exponential functions for data fitting. Experimental scheme as illustrated by Figure 7D. Details of evaluation are given by Table 2.

\section{Impact of Imipridone Derivatives}

Thus far, our results indicate that the concentration used for anti-tumor therapy and effective sodium channel-blocking activity of TIC10 (throughout used for TIC10 angular $_{\text {) happen }}$ in a similar concentration range (a weekly oral dose of $625 \mathrm{mg}$ TIC10 causes a maximal plasma concentration of $4.3 \mu \mathrm{M}$ (Allen et al., 2016)). To investigate whether there might be a correlation between these two properties, we tested two other derivatives, which were either inactive or more potent than TIC10 concerning their anti-tumor activity. These were $\mathrm{TIC} 0_{\text {linear }}$, an isomer of TIC10, which is completely inactive and ONC212, a fluorinated derivative which is at least 10 times more potent than TIC10 (Lev et al., 2017; Wagner et al., 2017; Nii et al., 2019). Our data clearly demonstrated that ONC212 has about the same potency as TIC10 angular while TIC10 $0_{\text {linear }}$ was less effective, especially in the protocol analyzing for slow inactivation. This poses the question, which property of the sodium channel is most important for its ability to support tumor cells. So far, the persistent sodium current is regarded as most important (Djamgoz et al., 2019). In this case all imipridone derivatives are expected to be equally effective as they suppress the "persistent current" with almost identical efficacy. However, this is not reflected by the anti-tumor activity of the different imipridone derivatives. Clearly, it must be taken into account that the anti-tumor activity refers to the ability of a drug to induce TRAIL, leading to cell death or affecting cell proliferation, whereas the blockade of sodium channels targets migration and invasion of tumor cells but not proliferation (Wagner et al., 2017). In this context, it is

TABLE 2 | Time-dependent interaction of imipridone derivatives with the inactivated state of the $\mathrm{hNa}_{\mathrm{v}} 1.5$

\begin{tabular}{lcccc}
\hline & ONC212 & Amount & TIC10 linear & Amount \\
\hline $2.5 \mu \mathrm{M}$ & & & & \\
$\tau_{1}(\mathrm{~s})$ & $0.2 \pm 0.06$ & $8.8 \pm 4.5 \%$ & $0.2 \pm 0.02$ & $10.6 \pm 3.1 \%$ \\
$\tau_{2}(\mathrm{~s})$ & $4.6 \pm 0.95$ & $57.4 \pm 6.6 \%$ & $6.7 \pm 1.24$ & $21.6 \pm 4.5 \%$ \\
$10 \mu \mathrm{M}$ & & & & \\
$\tau_{1}(\mathrm{~s})$ & $0.1 \pm 0.03$ & $45.2 \pm 6.5 \%$ & $0.1 \pm 0.02$ & $29.7 \pm 9.7 \%$ \\
$\tau_{2}(\mathrm{~s})$ & $2.2 \pm 0.27$ & $46.9 \pm 7.2 \%$ & $5.0 \pm 1.14$ & $37.4 \pm 4.9 \%$
\end{tabular}



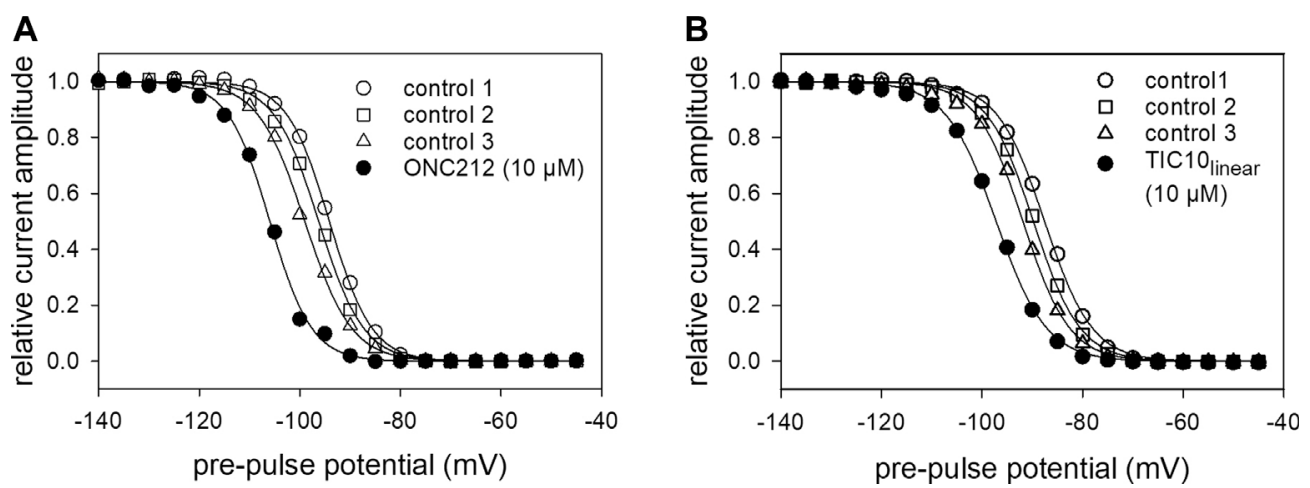

FIGURE 16 | Impact of imipridones derivatives on fast inactivation. Data of normalized transient currents in the absence (open symbols) and presence of drug (filled circles) are plotted against the pre-pulse potential. Experimental scheme as in Figure 5B. (A) ONC212 shifted inactivation midpoints by $-4.7 \pm 0.42 \mathrm{mV}$. (B) TIC10 linear shifted inactivation midpoints by $-3.0 \pm 0.37 \mathrm{mV}$. Data are corrected for the endogenous shift, estimated from three preceding controls.
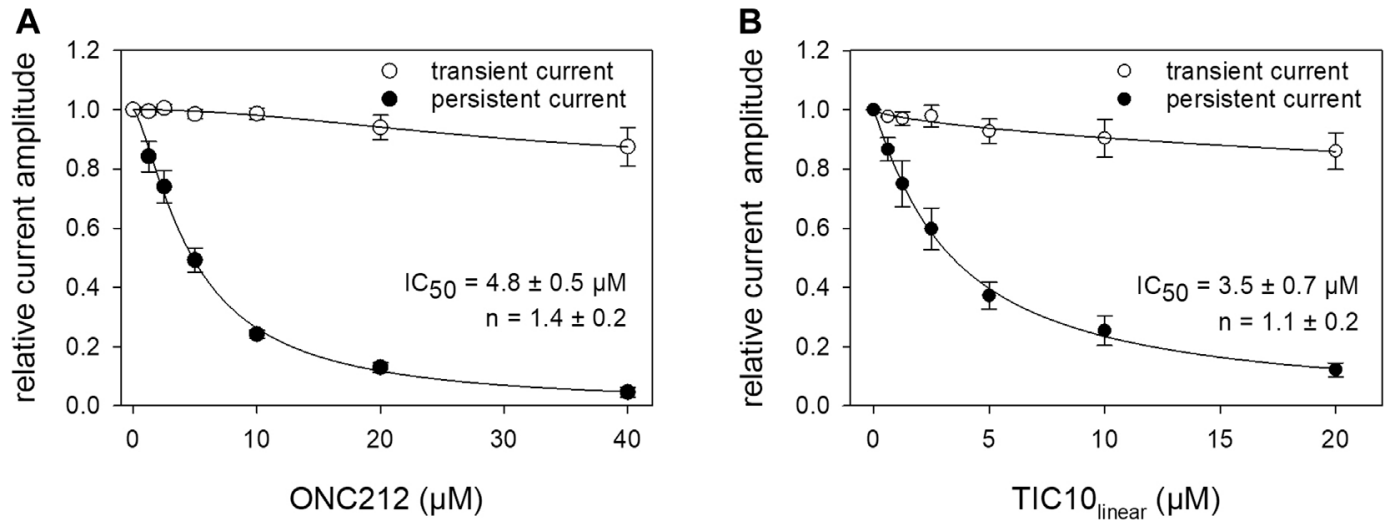

FIGURE 17 | Interaction of imipridone derivatives with the open state. The interaction with the open state was analyzed by means of the WCW mutant. Experimental scheme as illustrated by Figure 12A. Relative transient and persistent current amplitudes are given in relation to the concentration of ONC212 (A) or TIC10 ${ }_{\text {linear }}$ (B)

noteworthy that sodium channel blockers cannot completely suppress invasion. If sodium channels are totally inhibited, invasiveness is reduced only by about $35 \%$. Another point is that probably other channels/transporters contribute to an increase in intracellular sodium concentration (Leslie et al., 2019). Evidently, in these cases, invasiveness cannot be suppressed by blockers of VGSCs (Driffort et al., 2014; Roger et al., 2015). This also applies to tumor cells, which do not express VGSCs. Finally, even though imipridones affect different aspects of tumor progression, they do not eradicate tumors (Wang and Dougan, 2019).

\section{VGSCs Blocker and Cancer}

The discovery of an unexpected anti-tumor activity of a drug often starts with the observation of an obviously diminished incidence for cancer under a selected medication. A prominent example here is metformin, commonly used for treating diabetes type 2, for which the anti-tumor properties have been confirmed by many retrospective analysis (Evans et al.,
2005; Libby et al., 2009). Similarly, the outcome from the pharmacological treatment of neurological disorders has been analyzed. From most investigations, a positive correlation with respect to a beneficial outcome is reported (Reddy et al., 2015; Zhuo et al., 2019). However, there are also inverse associations (Takada et al., 2016). As many of the above-mentioned drugs operate as blockers for VGSCs, well-known blockers of this class are under investigation in different clinical studies for purposes of drug repurposing (Leslie et al., 2019). The list includes lidocaine, valproate, bupivacaine, or ranolazine. Interestingly, some of them preferentially target the persistent current (INaP) (Taverna et al., 1998; Wang et al., 2008). Beside this, the search for new blockers of VGSCs with respect to its potential application as a supplement in the tumor therapy is ongoing (Wang and Dougan, 2019). However, it should be kept in mind that beyond VGSCs many other ion channels and transporters might be of importance as supplements in tumor therapy (Cuddapah and Sontheimer, 2011; Biasiotta et al., 2016; Prevarskaya et al., 2018). 


\section{CONCLUSION}

TIC10 is a potent blocker of $\mathrm{hNa}_{\mathrm{v}} 1.5$, an important VGSC. Halfmaximal effects occur well within the concentration range used for tumor therapy. Thus, blockade of VGSCs might contribute to the anti-tumor activity of TIC10.

\section{DATA AVAILABILITY STATEMENT}

The raw data supporting the conclusions of this article will be made available by the authors, without undue reservation.

\section{AUTHOR CONTRIBUTIONS}

Conceptualization: KF; Formal analysis: EF, DM, MF, TZ, and KF; Funding acquisition: BJ and KF; Investigation: EF,

\section{REFERENCES}

Allen, J. E., Kline, C. L., Prabhu, V. V., Wagner, J., Ishizawa, J., Madhukar, N., et al. (2016). Discovery and Clinical Introduction of First-In-Class Imipridone ONC201. Oncotarget 7, 74380-74392. doi:10.18632/oncotarget.11814

Allen, J. E., Krigsfeld, G., Mayes, P. A., Patel, L., Dicker, D. T., Patel, A. S., et al. (2013). Dual Inactivation of Akt and ERK by TIC10 Signals Foxo3a Nuclear Translocation, TRAIL Gene Induction, and Potent Antitumor Effects. Sci. Transl. Med. 5, 171ra17. doi:10.1126/scitranslmed.3004828

Anderson, P. M., and Scott, J. (2017). Imipridone Family on Successful TRAIL. Cell Cycle 16, 1487-1488. doi:10.1080/15384101.2017.1345237

Arrillaga-Romany, I., Odia, Y., Prabhu, V. V., Tarapore, R. S., Merdinger, K., Stogniew, M., et al. (2020). Biological Activity of Weekly ONC201 in Adult Recurrent Glioblastoma Patients. Neuro. Oncol. 22, 94-102. doi:10.1093/ neuonc/noz164

Bean, B. P., Cohen, C. J., and Tsien, R. W. (1983). Lidocaine Block of Cardiac Sodium Channels. J. Gen. Physiol. 81, 613-642. doi:10.1085/jgp.81.5.613

Belardinelli, L., Liu, G., Smith-Maxwell, C., Wang, W. Q., El-Bizri, N., Hirakawa, R., et al. (2013). A Novel, Potent, and Selective Inhibitor of Cardiac Late Sodium Current Suppresses Experimental Arrhythmias. J. Pharmacol. Exp. Ther. 344, 23-32. doi:10.1124/jpet.112.198887

Biasiotta, A., D’Arcangelo, D., Passarelli, F., Nicodemi, E. M., and Facchiano, A. (2016). Ion Channels Expression and Function Are Strongly Modified in Solid Tumors and Vascular Malformations. J. Transl. Med. 14, 285. doi:10.1186/ s12967-016-1038-y

Brahimi-Horn, M. C., and Pouysségur, J. (2007). Oxygen, a Source of Life and Stress. FEBS Lett. 581, 3582-3591. doi:10.1016/j.febslet.2007.06.018

Carter, B. C., and Bean, B. P. (2009). Sodium Entry during Action Potentials of Mammalian Neurons: Incomplete Inactivation and Reduced Metabolic Efficiency in Fast-Spiking Neurons. Neuron 64, 898-909. doi:10.1016/ j.neuron.2009.12.011

Catterall, W. A., Goldin, A. L., and Waxman, S. G. (2005). International Union of Pharmacology. XLVII. Nomenclature and Structure-Function Relationships of Voltage-Gated Sodium Channels. Pharmacol. Rev. 57, 397-409. doi:10.1124/ pr.57.4.4

Catterall, W. A. (2014). Structure and Function of Voltage-Gated Sodium Channels at Atomic Resolution. Exp. Physiol. 99, 35-51. doi:10.1113/ expphysiol.2013.071969

Cuddapah, V. A., and Sontheimer, H. (2011). Ion Channels and Transporters [corrected] in Cancer. 2. Ion Channels and the Control of Cancer Cell Migration. Am. J. Physiol. Cel. Physiol 301, C541-C549. doi:10.1152/ ajpcell.00102.2011
GK, and KF; Supervision: KF; Validation: EF, DM, GK, MF, TZ, and KF; Visualization: MF, KF; Writing-original draft: $\mathrm{DM}$, and KF; Writing-reviewing and editing: All authors. All authors have read and agreed to the published version of the article.

\section{FUNDING}

The work was supported by the Department of Anesthesiology and Intensive Care Medicine, University Hospital of Ulm, Germany.

\section{ACKNOWLEDGMENTS}

We thank Margot Autenrieth-Kronenthaler and Karin Schoknecht for excellent technical assistance.

Djamgoz, M. B., and Onkal, R. (2013). Persistent Current Blockers of VoltageGated Sodium Channels: a Clinical Opportunity for Controlling Metastatic Disease. Recent Pat Anticancer Drug Discov. 8, 66-84. doi:10.2174/ 15748928130107

Djamgoz, M. B. A., Fraser, S. P., and Brackenbury, W. J. (2019). In Vivo Evidence for Voltage-Gated Sodium Channel Expression in Carcinomas and Potentiation of Metastasis. Cancers 11, 1675. doi:10.3390/cancers11111675

Driffort, V., Gillet, L., Bon, E., Marionneau-Lambot, S., Oullier, T., Joulin, V., et al. (2014). Ranolazine Inhibits NaV1.5-mediated Breast Cancer Cell Invasiveness and Lung Colonization. Mol. Cancer 13, 264. doi:10.1186/1476-4598-13-264

Evans, J. M., Donnelly, L. A., Emslie-Smith, A. M., Alessi, D. R., and Morris, A. D. (2005). Metformin and Reduced Risk of Cancer in Diabetic Patients. BMJ 330, 1304-1305. doi:10.1136/bmj.38415.708634.F7

Föhr, K. J., Knippschild, U., Herkommer, A., Fauler, M., Peifer, C., Georgieff, M., et al. (2017). State-dependent Block of Voltage-Gated Sodium Channels by the Casein-Kinase 1 Inhibitor IC261. Invest. New Drugs 35, 277-289. doi:10.1007/ s10637-017-0429-0

Föhr, K. J., Nastos, A., Fauler, M., Zimmer, T., Jungwirth, B., and Messerer, D. A. C. (2021). Block of Voltage-Gated Sodium Channels by Atomoxetine in a Stateand Use-dependent Manner. Front. Pharmacol. 12, 622489. doi:10.3389/ fphar.2021.622489

Fraser, S. P., Diss, J. K., Chioni, A. M., Mycielska, M. E., Pan, H., Yamaci, R. F., et al. (2005). Voltage-gated Sodium Channel Expression and Potentiation of Human Breast Cancer Metastasis. Clin. Cancer Res. 11, 5381-5389. doi:10.1158/10780432.CCR-05-0327

Grant, A. O., Chandra, R., Keller, C., Carboni, M., and Starmer, C. F. (2000). Block of Wild-type and Inactivation-Deficient Cardiac Sodium Channels IFM/QQQ Stably Expressed in Mammalian Cells. Biophys. J. 79, 3019-3035. doi:10.1016/ S0006-3495(00)76538-6

Grimes, J. A., Fraser, S. P., Stephens, G. J., Downing, J. E., Laniado, M. E., Foster, C. S., et al. (1995). Differential Expression of Voltage-Activated Na+ Currents in Two Prostatic Tumour Cell Lines: Contribution to Invasiveness In Vitro. FEBS Lett. 369, 290-294. doi:10.1016/0014-5793(95)00772-2

Hamill, O. P., Marty, A., Neher, E., Sakmann, B., and Sigworth, F. J. (1981). Improved Patch-Clamp Techniques for High-Resolution Current Recording from Cells and Cell-free Membrane Patches. Pflugers Arch. 391, 85-100. doi:10.1007/BF00656997

Ilyin, V. I., Hodges, D. D., Whittemore, E. R., Carter, R. B., Cai, S. X., and Woodward, R. M. (2005). V102862 (Co 102862): a Potent, Broad-Spectrum State-dependent Blocker of Mammalian Voltage-Gated Sodium Channels. Br. J. Pharmacol. 144, 801-812. doi:10.1038/sj.bjp.0706058

Ishida, C. T., Zhang, Y., Bianchetti, E., Shu, C., Nguyen, T. T. T., Kleiner, G., et al. (2018). Metabolic Reprogramming by Dual AKT/ERK Inhibition through 
Imipridones Elicits Unique Vulnerabilities in Glioblastoma. Clin. Cancer Res. 24, 5392-5406. doi:10.1158/1078-0432.CCR-18-1040

Ishizawa, J., Zarabi, S. F., Davis, R. E., Halgas, O., Nii, T., Jitkova, Y., et al. (2019). Mitochondrial ClpP-Mediated Proteolysis Induces Selective Cancer Cell Lethality. Cancer Cell 35, 721-e9. e9. doi:10.1016/j.ccell.2019.03.014

Ju, Y. K., Saint, D. A., and Gage, P. W. (1996). Hypoxia Increases Persistent Sodium Current in Rat Ventricular Myocytes. J. Physiol. 497 (Pt 2), 337-347. doi:10.1113/jphysiol.1996.sp021772

Kline, C. L., Van den Heuvel, A. P., Allen, J. E., Prabhu, V. V., Dicker, D. T., and ElDeiry, W. S. (2016). ONC201 Kills Solid Tumor Cells by Triggering an Integrated Stress Response Dependent on ATF4 Activation by Specific eIF2a Kinases. Sci. Signal. 9, ra18. doi:10.1126/scisignal.aac4374

Kline, C. L. B., Ralff, M. D., Lulla, A. R., Wagner, J. M., Abbosh, P. H., Dicker, D. T., et al. (2018). Role of Dopamine Receptors in the Anticancer Activity of ONC201. Neoplasia 20, 80-91. doi:10.1016/j.neo.2017.10.002

Leslie, T. K., James, A. D., Zaccagna, F., Grist, J. T., Deen, S., Kennerley, A., et al. (2019). Sodium Homeostasis in the Tumour Microenvironment. Biochim. Biophys. Acta Rev. Cancer 1872, 188304. doi:10.1016/ j.bbcan.2019.07.001

Lev, A., Lulla, A. R., Wagner, J., Dicker, D. T., and El-Deiry, W. S. (2017). Abstract 1067: Anti-cancer Efficacy of Imipridones in Pancreatic Cancer: Single Agent ONC212 or Combination of ONC201 with IGF1-R Inhibition. Cancer Res., 1067. doi:10.1158/1538-7445.AM2017-1067

Li, L., Li, P., Fang, J., Li, Q., Xiao, H., Zhou, H., et al. (2015). Simultaneous Quantitation of $\mathrm{Na}(+)$ and $\mathrm{K}(+)$ in Single Normal and Cancer Cells Using a New Near-Infrared Fluorescent Probe. Anal. Chem. 87, 6057-6063. doi:10.1021/acs.analchem.5b00571

Libby, G., Donnelly, L. A., Donnan, P. T., Alessi, D. R., Morris, A. D., and Evans, J. M. (2009). New Users of Metformin Are at Low Risk of Incident Cancer: a Cohort Study Among People with Type 2 Diabetes. Diabetes Care 32, 1620-1625. doi:10.2337/dc08-2175

Ludolph, A. G., Udvardi, P. T., Schaz, U., Henes, C., Adolph, O., Weigt, H. U., et al. (2010). Atomoxetine Acts as an NMDA Receptor Blocker in Clinically Relevant Concentrations. Br. J. Pharmacol. 160, 283-291. doi:10.1111/ j.1476-5381.2010.00707.x

Ma, Z., Gao, G., Fang, K., and Sun, H. (2019). Development of Novel Anticancer Agents with a Scaffold of Tetrahydropyrido[4,3-D]pyrimidine-2,4-Dione. ACS Med. Chem. Lett. 10, 191-195. doi:10.1021/acsmedchemlett.8b00531

Maltsev, V. A., Sabbah, H. N., Higgins, R. S., Silverman, N., Lesch, M., and Undrovinas, A. I. (1998). Novel, Ultraslow Inactivating Sodium Current in Human Ventricular Cardiomyocytes. Circulation 98, 2545-2552. doi:10.1161/ 01.cir.98.23.2545

McNulty, M. M., Kyle, J. W., Lipkind, G. M., and Hanck, D. A. (2006). An Inner Pore Residue (Asn406) in the Nav1.5 Channel Controls Slow Inactivation and Enhances Mibefradil Block to T-type Ca2+ Channel Levels. Mol. Pharmacol. 70, 1514-1523. doi:10.1124/mol.106.027177

Nii, T., Prabhu, V. V., Ruvolo, V., Madhukar, N., Zhao, R., Mu, H., et al. (2019). Imipridone ONC212 Activates Orphan G Protein-Coupled Receptor GPR132 and Integrated Stress Response in Acute Myeloid Leukemia. Leukemia 33, 2805-2816. doi:10.1038/s41375-019-0491-z

Noble, D., and Noble, P. J. (2006). Late Sodium Current in the Pathophysiology of Cardiovascular Disease: Consequences of Sodium-Calcium Overload. Heart 92, iv1-iv5. doi:10.1136/hrt.2005.078782

Onkal, R., and Djamgoz, M. B. (2009). Molecular Pharmacology of Voltage-Gated Sodium Channel Expression in Metastatic Disease: Clinical Potential of Neonatal Nav1.5 in Breast Cancer. Eur. J. Pharmacol. 625, 206-219. doi:10.1016/j.ejphar.2009.08.040

Ouwerkerk, R., Jacobs, M. A., Macura, K. J., Wolff, A. C., Stearns, V., Mezban, S. D., et al. (2007). Elevated Tissue Sodium Concentration in Malignant Breast Lesions Detected with Non-invasive 23Na MRI. Breast Cancer Res. Treat. 106, 151-160. doi:10.1007/s10549-006-9485-4

Patino, G. A., and Isom, L. L. (2010). Electrophysiology and beyond: Multiple Roles of Na+ Channel $\beta$ Subunits in Development and Disease. Neurosci. Lett. 486, 53-59. doi:10.1016/j.neulet.2010.06.050

Poulin, H., Bruhova, I., Timour, Q., Theriault, O., Beaulieu, J. M., Frassati, D., et al. (2014). Fluoxetine Blocks Nav1.5 Channels via a Mechanism Similar to that of Class 1 Antiarrhythmics. Mol. Pharmacol. 86, 378-389. doi:10.1124/ mol.114.093104
Prevarskaya, N., Skryma, R., and Shuba, Y. (2018). Ion Channels in Cancer: Are Cancer Hallmarks Oncochannelopathies. Physiol. Rev. 98, 559-621. doi:10.1152/physrev.00044.2016

Pruss, M., Dwucet, A., Tanriover, M., Hlavac, M., Kast, R. E., Debatin, K. M., et al. (2020). Dual Metabolic Reprogramming by ONC201/TIC10 and 2Deoxyglucose Induces Energy Depletion and Synergistic Anti-cancer Activity in Glioblastoma. Br. J. Cancer 122, 1146-1157. doi:10.1038/s41416020-0759-0

Reddy, J. P., Dawood, S., Mitchell, M., Debeb, B. G., Bloom, E., Gonzalez-Angulo, A. M., et al. (2015). Antiepileptic Drug Use Improves Overall Survival in Breast Cancer Patients with Brain Metastases in the Setting of Whole Brain Radiotherapy. Radiother. Oncol. 117, 308-314. doi:10.1016/ j.radonc.2015.10.009

Roger, S., Gillet, L., Le Guennec, J. Y., and Besson, P. (2015). Voltage-gated Sodium Channels and Cancer: Is Excitability Their Primary Role. Front. Pharmacol. 6, 152. doi:10.3389/fphar.2015.00152

Roger, S., Le Guennec, J. Y., and Besson, P. (2004). Particular Sensitivity to Calcium Channel Blockers of the Fast Inward Voltage-dependent Sodium Current Involved in the Invasive Properties of a Metastastic Breast Cancer Cell Line. Br. J. Pharmacol. 141, 610-615. doi:10.1038/sj.bjp.0705649

Stein, M. N., Malhotra, J., Tarapore, R. S., Malhotra, U., Silk, A. W., Chan, N., et al. (2019). Safety and Enhanced Immunostimulatory Activity of the DRD2 Antagonist ONC201 in Advanced Solid Tumor Patients with Weekly Oral Administration. J. Immunother. Cancer 7, 136. doi:10.1186/s40425019-0599-8

Takada, M., Fujimoto, M., Motomura, H., and Hosomi, K. (2016). Inverse Association between Sodium Channel-Blocking Antiepileptic Drug Use and Cancer: Data Mining of Spontaneous Reporting and Claims Databases. Int. J. Med. Sci. 13, 48-59. doi:10.7150/ijms.13834

Taverna, S., Mantegazza, M., Franceschetti, S., and Avanzini, G. (1998). Valproate Selectively Reduces the Persistent Fraction of $\mathrm{Na}+$ Current in Neocortical Neurons. Epilepsy Res. 32, 304-308. doi:10.1016/s0920-1211(98)00060-6

Theile, J. W., and Cummins, T. R. (2011). Recent Developments Regarding Voltage-Gated Sodium Channel Blockers for the Treatment of Inherited and Acquired Neuropathic Pain Syndromes. Front. Pharmacol. 2, 54. doi:10.3389/ fphar.2011.00054

Urbani, A., and Belluzzi, O. (2000). Riluzole Inhibits the Persistent Sodium Current in Mammalian CNS Neurons. Eur. J. Neurosci. 12, 3567-3574. doi:10.1046/ j.1460-9568.2000.00242.x

Wagner, J., Kline, C. L., Ralff, M. D., Lev, A., Lulla, A., Zhou, L., et al. (2017). Preclinical Evaluation of the Imipridone Family, Analogs of Clinical Stage Anticancer Small Molecule ONC201, Reveals Potent Anti-cancer Effects of ONC212. Cell Cycle 16, 1790-1799. doi:10.1080/15384101.2017.1325046

Walzik, S., Schroeter, A., Benndorf, K., and Zimmer, T. (2011). Alternative Splicing of the Cardiac Sodium Channel Creates Multiple Variants of Mutant T1620K Channels. PLoS ONE 6, e19188. doi:10.1371/journal.pone.0019188

Wang, G. K., Calderon, J., and Wang, S. Y. (2008). State- and Use-dependent Block of Muscle Nav1.4 and Neuronal Nav1.7 Voltage-Gated Na+Channel Isoforms by Ranolazine. Mol. Pharmacol. 73, 940-948. doi:10.1124/ mol.107.041541

Wang, G. K., Russell, C., and Wang, S. Y. (2004). Mexiletine Block of Wild-type and Inactivation-Deficient Human Skeletal Muscle hNav1.4 Na+ Channels. J. Physiol. 554, 621-633. doi:10.1113/ jphysiol.2003.054973

Wang, G. K., Russell, C., and Wang, S. Y. (2003). State-dependent Block of Wildtype and Inactivation-Deficient $\mathrm{Na}+$ Channels by Flecainide. J. Gen. Physiol. 122, 365-374. doi:10.1085/jgp.200308857

Wang, G. K., Russell, G., and Wang, S. Y. (2013). Persistent Human Cardiac Na+ Currents in Stably Transfected Mammalian Cells: Robust Expression and Distinct Open-Channel Selectivity Among Class 1 Antiarrhythmics. Channels (Austin) 7, 263-274. doi:10.4161/chan.25056

Wang, S., and Dougan, D. A. (2019). The Direct Molecular Target for Imipridone ONC201 Is Finally Established. Cancer Cell 35, 707-708. doi:10.1016/ j.ccell.2019.04.010

Yamaci, R. F., Fraser, S. P., Battaloglu, E., Kaya, H., Erguler, K., Foster, C. S., et al. (2017). Neonatal Nav1.5 Protein Expression in normal Adult Human Tissues and Breast Cancer. Pathol. Res. Pract. 213, 900-907. doi:10.1016/ j.prp.2017.06.003 
Zhuo, C., Xun, Z., Hou, W., Ji, F., Lin, X., Tian, H., et al. (2019). Surprising Anticancer Activities of Psychiatric Medications: Old Drugs Offer New Hope for Patients with Brain Cancer. Front. Pharmacol. 10, 1262. doi:10.3389/ fphar.2019.01262

Conflict of Interest: The authors declare that the research was conducted in the absence of any commercial or financial relationships that could be construed as a potential conflict of interest.

Publisher's Note: All claims expressed in this article are solely those of the authors and do not necessarily represent those of their affiliated organizations, or those of the publisher, the editors and the reviewers. Any product that may be evaluated in this article, or claim that may be made by its manufacturer, is not guaranteed or endorsed by the publisher.

Copyright $\odot 2021$ Fuchs, Messerer, Karpel-Massler, Fauler, Zimmer, Jungwirth and Föhr. This is an open-access article distributed under the terms of the Creative Commons Attribution License (CC BY). The use, distribution or reproduction in other forums is permitted, provided the original author(s) and the copyright owner(s) are credited and that the original publication in this journal is cited, in accordance with accepted academic practice. No use, distribution or reproduction is permitted which does not comply with these terms. 